\title{
Spectrum Pricing Games with Spatial Reuse in Cognitive Radio Networks
}

\author{
Gaurav S. Kasbekar and Saswati Sarkar
}

\begin{abstract}
In Cognitive Radio Networks (CRN), there are multiple primary and secondary users in a region, and primaries can lease out their unused bandwidth to secondaries in exchange for a fee. This gives rise to price competition among the primaries, wherein each primary tries to attract secondaries by setting a lower price for its bandwidth than the other primaries. Radio spectrum has the distinctive feature that transmissions at neighboring locations on the same channel interfere with each other, whereas the same channel can be used at far-off locations without mutual interference. So in the above price competition scenario in a CRN, each primary must jointly select a set of mutually non-interfering locations within the region (which corresponds to an independent set in the conflict graph representing the region) at which to offer bandwidth and the price at each location. In this paper, we analyze this price competition scenario as a game and seek a Nash Equilibrium (NE). We identify a class of conflict graphs, which we refer to as mean valid graphs, such that the conflict graphs of a large number of topologies that commonly arise in practice are mean valid. We explicitly compute a symmetric NE in mean valid graphs and show that it is unique.
\end{abstract}

Index Terms-Cognitive Radio Networks, Price Competition, Game Theory, Nash Equilibrium, Spatial Reuse, Mean Valid Graphs

\section{INTRODUCTION}

Cognitive Radio Networks (CRN) [1] are emerging as a promising solution for the efficient use of spectrum. In these networks, there are two types of spectrum users: (i) primary users who lease certain portions (channels or bands) of the spectrum directly from the regulator, and (ii) secondary users who can use a channel when it is not used by the primary. We consider a CRN with multiple primary and secondary users in a region. Time is slotted, and in every slot, each primary has unused bandwidth with some probability, which it would like to lease to the secondaries. Now, secondaries would like to lease bandwidth from the primaries that offer it at a low price, which results in price competition among the primaries. Price competition is naturally modeled using game theory [2], and has been extensively studied in economics using, for example, the classical Bertrand game [5] and its variants.

However, a CRN has several distinguishing features, which makes the price competition very different from oligopolies encountered in economics. First, in every slot, each primary may or may not have unused bandwidth available. So a primary who has unused bandwidth is uncertain about the number of primaries from whom it will face competition.

G. Kasbekar and S. Sarkar are with the Department of Electrical and Systems Engineering at University of Pennsylvania, Philadelphia, PA, U.S.A. Their email addresses are kgaurav@seas.upenn.edu and swati@seas.upenn.edu respectively.

Part of this paper was presented at MobiHoc'10.
Setting a low price will result in unnecessarily low revenues in the event that very few other primaries have unused bandwidth, because even with a higher price the primary's bandwidth would have been bought, and vice versa. Second, spectrum is a commodity that allows spatial reuse: the same band can be simultaneously used at far-off locations without interference; on the other hand, simultaneous transmissions at neighboring locations on the same band interfere with each other. Thus, spatial reuse provides an opportunity to primaries to increase their profit by selling the same band to secondaries at different locations, which they can utilize subject to satisfying the interference constraints. So when multiple primaries own bandwidth in a large region, each needs to decide on a set of non-interfering locations within the region, which corresponds to an independent set in the conflict graph representing the region, at which to offer bandwidth. This is another source of strategic interaction among the primaries- each primary would like to select a maximum-sized independent set to offer bandwith at; but if a lot of primaries offer bandwidth at the same locations, there is intense competition at those locations. So a primary would have benefited by instead offering bandwidth at a smaller independent set and charging high prices at those locations.

Some progress has been made in addressing the issue of bandwidth uncertainty, both in the CRN setting [19], [20] and in the context of price competition among multiple firms in the Economics literature [8]. The issue of spatial reuse, however, arises specifically in the context of spectrum trading and has not been investigated either in prior work on price competition in CRNs [11], [12], [13], [14], [15], [16] or otherwise. See Section II for a survey of related work.

In this paper, we analyze price competition in CRNs jointly considering both bandwidth uncertainty and spatial reuse, and specifically focusing on the latter. We formulate the problem as a game in which each primary needs to select (i) a set of locations at which to offer bandwidth and (ii) the price of bandwidth at each location. We seek to obtain a Nash Equilibrium (NE) in this game. The challenge in doing so is that, since prices can take values from a continuous set, the strategy sets of primaries are uncountably infinite. So it is not apriori clear whether a NE exists, and there is no standard algorithm for finding a NE, unlike when each player's strategy set is finite [2].

We focus on symmetric NE, in which every player uses the same strategy, since our game turns out to be a symmetric game. Our first contribution is to prove a separation theorem (Section III-D), which states that in a symmetric NE, the price distributions used by the primaries at different nodes are uniquely determined once the independent set selection 
distributions are obtained. We therefore focus on computing the latter, which in turn provides the joint independent set and price selection strategies, by virtue of our prior results [19], [20] that characterize price competition in the absence of spatial reuse.

We focus on a class of conflict graphs that we refer to as mean valid graphs. These are graphs whose node set can be partitioned into $d$ disjoint maximal independent sets $I_{1}, \ldots, I_{d}$, for some integer $d \geq 2$, and which satisfy another technical condition to be introduced later (in Section IV). As we show in Section VI-A, it turns out that the conflict graphs of a large number of topologies that arise in practice are mean valid. In particular, several lattice arrangements of nodes in two and three dimensions are mean valid, e.g., a grid graph in two dimensions, such as that in part (b) of Fig. 3 or Fig. 13, which may be the conflict graph of shops in a shopping complex, the conflict graph of a cellular network with hexagonal cells (see Figs. 7 and 8), a grid graph in three dimensions, which may represent offices in a corporate building (see Fig. 6) etc.

We show that a mean valid graph has a unique symmetric $\mathrm{NE}$; in this NE, each primary offers bandwidth only at some or all of the independent sets in $I_{1}, \ldots, I_{d}$ with positive probability and with 0 probability at every other independent set. These probabilities (and thereby the NE strategies) can be explicitly computed by solving a system of equations that we provide. The fact that primaries offer bandwidth with a positive probability at only a small number of independent sets is a surprising result, because in most graphs, including the examples in the previous paragraph, the number of independent sets is exponential in the number of nodes. Our characterization of the symmetric NE also reveals that when the probability $q$ that a primary has unused bandwidth is small, primaries only offer bandwidth at the larger independent sets out of $I_{1}, \ldots, I_{d}$ and as $q$ increases, primaries also start offering bandwidth at the smaller ones. This is because, for given prices, a larger independent set yields a larger revenue. However, as $q$ increases, the price competition at the large independent sets becomes intense and drives down the prices and revenues at those independent sets. So primaries also offer bandwidth at the smaller independent sets.

The paper is organized as follows. We review related work in Section II and describe our model in Section III-A. In Section III-C, we provide a summary of the results on price competition at a single location that were developed in our prior work [19], [20]. We introduce mean valid graphs in Section IV and provide several examples. In Section V, we prove the theorem, discussed above, on characterization of the unique symmetric NE in mean valid graphs. In Section VI, we show that the conflict graphs of several topologies of practical interest as well as some other common types of graphs are mean valid. In Section VII-A, we show that the mean validity condition is a necessary condition for the existence of a symmetric NE of the above form when $d=2$, and also find the symmetric NE and prove its uniqueness in a specific non mean valid graph.

\section{RELATED WORK}

Pricing related issues have been extensively studied in the context of wired networks and the Internet; see [10] for an overview. Price competition among spectrum providers in wireless networks has been studied in [11], [12], [13], [14], [15], [16]. Specifically, Niyato et. al. analyze price competition among multiple primaries in CRNs [15], [16]. However, neither uncertain bandwidth availability, nor spatial reuse is modeled in any of the above papers. Also, most of these papers do not explicitly find a NE (exceptions are [12], [15]). Our model incorporates both uncertain bandwidth availability and spatial reuse, which makes the problem challenging; despite this, we are able to explicitly compute a NE. Zhou et. al. [17] have designed double auction based spectrum trades in which an auctioneer chooses an allocation taking into account spatial reuse and bids. However, in the price competition model we consider, each primary independently sells bandwidth, and hence a central entity such as an auctioneer is not required.

In the economics literature, the Bertand game has been traditionally used to study price competition in oligopolies [5]in this game, each seller quotes a price for a good, and the buyers buy from the seller that quotes the lowest price. Several variants of the Bertrand game have also been studied, e.g., [6], [7], [8], [18]. Chawla et al. [18] consider price competition in networks where each seller owns a capacityconstrained link, and decides the price for using it; the consumers choose paths they would use in the networks based on the prices declared and pay the sellers accordingly. Note that in our model, the sellers need to decide the locations at which to offer bandwidth as well as the price at each location. In addition, the link capacities are deterministic in [18], whereas the availability of bandwidth is random in our model. However, none of the above papers [6], [7], [8], [18] consider the spectrum-specific issue of spatial reuse, which introduces a new dimension, that each player not only needs to determine the price of the commodity it owns (as in [6], [7], [8], [18]), but also select an independent set to compete in. The joint decision problem significantly complicates the analysis.

\section{Model, Problem Definition And BACKGRound}

\section{A. Model}

Suppose there are $n \geq 2$ primaries, each of whom owns a channel throughout a large region. Time is divided into slots of equal duration. In every slot, each primary independently either uses its channel throughout the region to satisfy its own subscriber demand, or does not use it anywhere in the region. A typical scenario where this happens is when primaries broadcast the same signal over the entire region, e.g., if they are television broadcasters. Let $q \in(0,1)$ be the probability that a primary does not use its channel in a slot (to satisfy its subscriber demand). For tractability, we assume that the probability $q$ is the same for all primaries; we discuss the effect of relaxing this assumption in Section IX. Now, the region contains smaller parts, which we refer to as locations. For example, the large region may be a state, and the locations may be towns within it. We first assume that there are $k$ secondaries 
at each location, and in Section VII-B outline how the results can be generalized to allow for random and potentially unequal number of secondaries at different locations.

A primary who has unused bandwidth in a slot can lease it out to secondaries at a subset of the locations, provided this subset satisfies the spatial reuse constraints, which we describe next. The overall region can be represented by an undirected graph [4] $G=(V, E)$, where $V$ is the set of nodes and $E$ is the set of edges, called the conflict graph, in which each node represents a location, and there is an edge between two nodes iff transmissions at the corresponding locations interfere with each other. Recall that an independent set [4] (I.S.) in a graph is a set of nodes such that there is no edge between any pair of nodes in the set. Now, a primary who is not using its channel must offer it at a set of mutually non-interfering locations, or equivalently, at an I.S. of nodes; otherwise secondaries ${ }^{1}$ will not be able to successfully transmit simultaneously using the bandwidth they purchase, owing to mutual interference.

A primary $i$ who offers bandwidth at an I.S. $I$, must also determine for each node $v \in I$, the access fee, $p_{i, v}$, to be charged to a secondary if the latter leases the bandwidth at node $v$. A primary incurs a cost of $c \geq 0$ per slot per node for leasing out bandwidth. This cost may arise, for example, if the secondary uses the primary's infrastructure to access the Internet.

We assume that $p_{i, v} \leq \nu$ for each primary $i$ and each node $v$, for some constant $\nu>c$. This upper bound $\nu$ may arise as follows:

1) The spectrum regulator may impose this upper bound to ensure that primaries do not excessively overprice bandwidth even when competition is limited owing to bandwidth scarcity or high demands from secondaries, or when the primaries collude.

2) Alternatively, the valuation of each secondary for 1 unit of bandwidth may be $\nu$, and no secondary will buy bandwidth at a price that exceeds its valuation.

We assume that the primaries know this upper limit $\nu$.

Secondaries buy bandwidth from the primaries that offer the lowest price. More precisely, in a given slot, let $Z$ be the number of primaries who offer unused bandwidth at a node. Then, since there are $k$ secondaries at each node, the bandwidth of the $\min (Z, k)$ primaries that offer the lowest prices is bought (ties are resolved at random) at the node. The utility of a primary $i$ who offers bandwidth at an I.S. $I$ and sets a price of $p_{i, v}$ at node $v \in I$ is given by $\sum\left(p_{i, v}-c\right)$, where the summation is over the nodes $v \in I$ at which primary $i$ 's bandwidth is bought. (The utility is 0 if bandwidth is not bought at any node).

Thus, each primary must jointly select an I.S. at which to offer bandwidth, and the prices to set at the nodes in it. Both the I.S. and price selection may be random. Thus, a strategy, say $\psi_{i}$, of a primary $i$ provides a probability mass function (p.m.f.) for selection among the I.S. and the price

\footnotetext{
${ }^{1}$ Note that secondaries are usually customers or local providers, and purchase bandwidth for communication (and not television broadcasts). Thus, two secondaries can not use the same band simultaneously at interfering locations.
}

distribution it uses at each node (both selections contingent on having unused bandwidth). Note that we allow a primary to use different (and arbitrary) price distributions for different nodes (and therefore allow, but do not require, the selection of different prices at different nodes), and arbitrary p.m.f. (i.e., discrete distributions) for selection among the different I.S.

The case $k \geq n$ is trivial: in this case, the strategy of offering bandwidth at a maximum-sized I.S. and setting the maximum price $\nu$ at every node in the I.S. maximizes the utility of each primary $i$ regardless of the strategies of the other primaries. This is because, since $k \geq n$, the number of buyers at every node is always greater than or equal to the number of sellers. So henceforth, we assume that $k \leq n-1$.

\section{B. Symmetric Nash Equilibrium}

The vector $\left(\psi_{1}, \ldots, \psi_{n}\right)$ of strategies of the primaries is called a strategy profile [5]. Let $\psi_{-i}=$ $\left(\psi_{1}, \ldots, \psi_{i-1}, \psi_{i+1}, \ldots, \psi_{n}\right)$ denote the vector of strategies of primaries other than $i$. Let $E\left\{u_{i}\left(\psi_{i}, \psi_{-i}\right)\right\}$ denote the expected utility of primary $i$ when it adopts strategy $\psi_{i}$ and the other primaries adopt $\psi_{-i}$.

Definition 1 (Nash Equilibrium (NE), Symmetric NE):

A Nash equilibrium (NE) is a strategy profile such that no player can improve its expected utility by unilaterally deviating from its strategy [5]. Thus, $\left(\psi_{1}^{*}, \ldots, \psi_{n}^{*}\right)$ is a NE if for each primary $i$ :

$$
E\left\{u_{i}\left(\psi_{i}^{*}, \psi_{-i}^{*}\right)\right\} \geq E\left\{u_{i}\left(\widetilde{\psi}_{i}, \psi_{-i}^{*}\right)\right\}, \forall \widetilde{\psi}_{i}
$$

A NE $\left(\psi_{1}^{*}, \ldots, \psi_{n}^{*}\right)$ is a symmetric $N E$ if all players play identical strategies under it, i.e., $\psi_{1}^{*}=\psi_{2}^{*}=\ldots=\psi_{n}^{*}$.

Equation (1) says that when players other than $i$ play $\psi_{-i}^{*}$, $\psi_{i}^{*}$ maximizes $i$ 's expected utility; $\psi_{i}^{*}$ is said to be its best response [5] to $\psi_{-i}^{*}$.

Now, note that in the game defined in Section III-A, all the $n$ players (primaries) have identical strategy sets and utility functions. Such a game is said to be a symmetric game. In a symmetric game, in practice, it is challenging to implement a NE that is not symmetric- the simple example of two primaries and a NE of $\left(\psi_{1}^{*}, \psi_{2}^{*}\right)$ elucidates the inherent complications in the current context. If $\psi_{1}^{*} \neq \psi_{2}^{*}$, then since the game is symmetric, $\left(\psi_{2}^{*}, \psi_{1}^{*}\right)$ also constitutes a NE. If player 1 knows that player 2 is playing $\psi_{2}^{*}$ ( $\psi_{1}^{*}$ respectively), it would choose the best response $\psi_{1}^{*}\left(\psi_{2}^{*}\right.$ respectively), but it can not know player 2's choice between the two options without explicitly coordinating with him, which is again ruled out due to the competition between the two. Under symmetric NE, all players play the same strategy, and thus this quandary is somewhat limited- symmetric NE has indeed been advocated for symmetric games by several game theorists [9]. The natural question now is whether there exists at least one symmetric $\mathrm{NE}$, and also whether there is a unique symmetric NE (only uniqueness will eliminate the above quandary). Note that some symmetric games are known to have multiple symmetric NE, e.g. the "Meeting in New York game" (see [5], p. 221).

We show the existence of a symmetric NE, by explicitly computing one, and prove that it is the unique symmetric NE in our context. 


\section{Single Location}

In this subsection, we briefly summarize the main results for price competition among multiple primaries and secondaries at a single location, which were dealt with in detail in [19], [20].

Since there is only one location, there are no spatial reuse constraints, and the strategy of a primary $i$ is a distribution function (d.f.) $\psi_{i}$ (.), which it uses to select the price.

Let

$$
w(q, n)=\sum_{i=k}^{n-1}\left(\begin{array}{c}
n-1 \\
i
\end{array}\right) q^{i}(1-q)^{n-1-i} .
$$

Since each primary independently has unused bandwidth with probability (w.p.) $q, w(q, n)$ is the probability that $k$ or more out of $n-1$ primaries have unused bandwidth. We will later use the following fact [19]:

Lemma 1: $w(q, n)$ is a strictly increasing function of $q$ for fixed $n$.

Now, let:

$$
\tilde{p}=\nu-w(q, n)(\nu-c) .
$$

In [19], we showed that in the price competition game at a single location, there is a unique symmetric NE in which each primary randomizes over the prices in the range $[\tilde{p}, v]$ using a continuous distribution function (d.f.) $\psi($.) (see Theorem 2 in [19]). Also, $\psi($.$) is strictly increasing on [\tilde{p}, v]$; in particular, every price in $[\tilde{p}, v]$ is a best response for each primary [19]. An explicit expression for $\psi($.$) has been computed in [19].$ Finally, under this symmetric NE, each primary receives an expected payoff of [19]:

$$
\tilde{p}-c=(\nu-c)(1-w(q, n))
$$

\section{A Separation Result}

Recall that a strategy of a primary consists of a p.m.f. over I.S. and price distributions at individual nodes. We now provide a separation framework from which the price distributions at individual nodes in a symmetric NE follow once the I.S. selection p.m.f.s are determined.

Let $\mathscr{I}$ be the set of all I.S. in $G$. For convenience, we assume that the empty I.S. $I_{\emptyset} \in \mathscr{I}$ and we allow a primary to offer bandwith at $I_{\emptyset}$, i.e. to not offer bandwidth at any node, with some probability. Consider a symmetric strategy profile under which each primary offers bandwidth at I.S. $I \in \mathscr{I}$ w.p. $\beta(I)$, where:

$$
\sum_{I \in \mathscr{I}} \beta(I)=1
$$

The probability, say $\alpha_{v}$, with which each primary offers bandwidth at a node $v \in V$ equals the sum of the probabilities associated with all the I.S. that contain the node, i.e.

$$
\alpha_{v}=\sum_{I \in \mathscr{I}: v \in I} \beta(I)
$$

Now, considering that each primary has unused bandwidth w.p. $q$, it offers it at node $v$ w.p. $q \alpha_{v}$. The price selection problem at each node $v$ is now equivalent to that for the single location case, the difference being that each primary

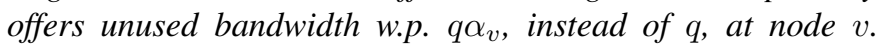
Thus:
Lemma 2: Suppose under a symmetric NE each primary selects node $v$ w.p. $\alpha_{v}$ if it has unused bandwidth. Then under that NE the price distribution of each primary at node $v$ is the d.f. $\psi($.$) in Section III-C, with q \alpha_{v}$ in place of $q$.

Thus, a symmetric NE strategy is completely specified once the I.S. selection p.m.f. $\{\beta(I): I \in \mathscr{I}\}$ (which will in turn provide the $\alpha_{v}$ s via (6)) is obtained.

\section{E. Node and I.S. Probabilities}

Consider a symmetric NE where each primary uses the strategy $\psi$, under which it offers bandwidth at I.S. $I \in \mathscr{I}$ with some probability $\beta(I)$. The probability, $\alpha_{v}$, with which each primary offers bandwidth at a node $v \in V$ is determined by the I.S. distribution $\{\beta(I): I \in \mathscr{I}\}$ via (6).

Now, for simplicity, we normalize $\nu-c=1$. With $w(q, n)$ as in (2), let:

$$
W(\alpha)=(1-w(q \alpha, n))(\nu-c)=(1-w(q \alpha, n)) .
$$

By Lemma 2, and similar to (4) in the single location case, in a symmetric NE if primaries offer bandwidth at a node with probability $\alpha$ (and play the single-node NE strategy with $q \alpha$ in place of $q$ at that node), then $W(\alpha)$ is the maximum expected payoff that each primary $i$ can get at that node. It gets this payoff $W(\alpha)$ if it sets any price in the range $[\nu-w(q \alpha, n)(\nu-c), \nu]$ at that node. Under the above symmetric NE with strategy profile $(\psi, \ldots, \psi)$, each primary offers bandwidth at node $v \in V$ w.p. $\alpha_{v}$. So the expected payoff of each primary $i$ is given by:

$$
E\left\{u_{i}\left(\psi, \psi_{-i}\right)\right\}=\sum_{v \in V} \alpha_{v} W\left(\alpha_{v}\right) .
$$

Now, in general, different I.S. distributions $\{\beta(I): I \in \mathscr{I}\}$ can result in the same node distribution ${ }^{2}\left\{\alpha_{v}: v \in V\right\}$. However, by (8), the expected payoff of each primary in a symmetric NE is completely determined by the node distribution, i.e. it is the same under different I.S. distributions that correspond to the same node distribution. So if primary $i$ knows the node distribution chosen by the other primaries, then it has sufficient information to choose its best response; it does not need to know their I.S. distribution in addition. Thus, the game aspect of the price competition, i.e. the strategic interaction between the primaries, is completely determined by the node distribution.

We now introduce a definition:

Definition 2 (Valid Distribution): An assignment $\left\{\alpha_{v}: v \in\right.$ $V\}$ of probabilities to the nodes is said to be a valid distribution if there exists a probability distribution $\{\beta(I): I \in \mathscr{I}\}$ such that for each $v \in V, \alpha_{v}=\sum_{I \in \mathscr{I}: v \in I} \beta(I)$.

Note that, given a valid distribution $\left\{\alpha_{v}: v \in V\right\}$, a corresponding I.S. distribution can be computed by solving the system of linear equations (6).

Thus, we can equivalently define the strategy of a primary in a symmetric NE as a node distribution $\left\{\alpha_{v}: v \in V\right\}$. So henceforth, we interchangeably speak of the strategy of a primary as either an I.S. distribution $\{\beta(I): I \in \mathscr{I}\}$ (note

\footnotetext{
${ }^{2}$ Although we refer to $\left\{\alpha_{v}: v \in V\right\}$ as a distribution, note that $\sum_{v \in V} \alpha_{v}$ need not equal 1 in general.
} 
that the price distribution follows by Lemma 2) or a node distribution $\left\{\alpha_{v}: v \in V\right\}$. Also, we say that the symmetric $\mathrm{NE}$ is unique if the node distribution $\left\{\alpha_{v}: v \in V\right\}$ is unique.

\section{MEAN VALid GRAPHS}

We now introduce a class of graphs, which we refer to as mean valid graphs. The motivation behind studying these graphs is that the conflict graphs of several topologies that commonly arise in practice are mean valid graphs. Also, as we show in the next section, these graphs have a unique symmetric NE, which can be explicitly computed and has a simple form.

\section{A. Definition}

Definition 3 (Mean Valid Graph): We refer to a graph $G=$ $(V, E)$ as mean valid if it satisfies the following two conditions:

1) Its vertex set can be partitioned into $d$ disjoint maximal ${ }^{3}$ I.S. for some integer $d \geq 2: V=I_{1} \cup I_{2} \cup \ldots \cup I_{d}$, where $I_{j}, j \in\{1, \ldots, d\}$, is a maximal I.S. and $I_{j} \cap I_{m}=\emptyset$, $j \neq m$.

Let $\left|I_{j}\right|=M_{j}$,

$$
M_{1} \geq M_{2} \geq \ldots \geq M_{d}
$$

and $I_{j}=\left\{a_{j, l}: l=1, \ldots, M_{j}\right\}$.

2) For every valid distribution ${ }^{4}$ in which a primary offers bandwidth at node $a_{j, l}$ w.p. $\alpha_{j, l}, j=1, \ldots, d$, $l=1, \ldots, M_{j}$,

$$
\sum_{j=1}^{d} \bar{\alpha}_{j} \leq 1
$$

where

$$
\bar{\alpha}_{j}=\frac{\sum_{l=1}^{M_{j}} \alpha_{j, l}}{M_{j}}, j \in\{1, \ldots, d\} .
$$

We now explain the two conditions in Definition 3. Recall that a graph $G=(V, E)$ is said to be $d$-partite if $V$ can be partitioned into $d$ disjoint I.S. $I_{1}, \ldots, I_{d}$ [4]. For example, when $d=2, G$ is a bipartite graph. The first condition in Definition 3 says that $G$ is a $d$-partite graph and has the additional property that each of $I_{1}, \ldots, I_{d}$ is a maximal I.S.

Now we explain Condition 2 in Definition 3 . Let $\left\{\alpha_{j, l}\right.$ : $\left.j=1, \ldots, d ; l=1, \ldots, M_{j}\right\}$ be an arbitrary valid distribution. Consider the distribution $\alpha_{j, l}^{\prime}=\bar{\alpha}_{j}$, with $\bar{\alpha}_{j}$ as in (11), i.e. for each $j$ and $l=1, \ldots, M_{j}, \alpha_{j, l}^{\prime}$ is set equal to the mean of $\alpha_{j, m}, m=1, \ldots, M_{j}$. If (10) is true, then this distribution of means is a valid distribution because it corresponds to the I.S. distribution $\left\{\beta\left(I_{j}\right)=\bar{\alpha}_{j}, j=1, \ldots, d, \beta\left(I_{\emptyset}\right)=\right.$ $\left.1-\sum_{j=1}^{d} \bar{\alpha}_{j} ; \beta(I)=0, I \neq I_{1}, \ldots, I_{d}, I_{\emptyset}\right\}$. Thus, Condition 2 in Definition 3 says that in $G$, the distribution of means corresponding to every valid distribution is valid. As we will see in Section $V$, this condition is the crux behind the fact that in the symmetric NE in a mean valid graph, each primary offers bandwidth with equal probabilities at all the nodes in $I_{j}$ for every $j=1, \ldots, d$.

${ }^{3}$ Recall that an I.S. $I$ is said to be maximal if $I \cup\{v\}$ is not an I.S. for all $v \in V$ [4].

${ }^{4}$ Note that we write $\alpha_{j, l}$ in place of $\alpha_{a_{j, l}}$ to simplify the notation.

\section{B. Examples}

Technical as Definition 3 may seem, it turns out that several conflict graphs that commonly arise in practice are mean valid. For example, consider the following graphs:

1) Let $\mathcal{G}_{m}$ denote a graph that is a linear arrangement of $m \geq 2$ nodes as shown in part (a) of Fig. 3, with an edge between each pair of adjacent nodes. As an example, this would be the conflict graph for locations along a highway or a row of roadside shops.

2) We consider two types of $m \times m$ grid graphs, denoted by $\mathcal{G}_{m, m}$ (see part (b) of Fig. 3) and $\mathcal{H}_{m, m}$ (see Fig. 13). In both these graphs, $m^{2}$ nodes (locations) are arranged in a square grid. In $\mathcal{G}_{m, m}$, there is an edge only between each pair of adjacent nodes in the same row or column. In $\mathcal{H}_{m, m}$, in addition to these edges, there are also edges between nodes that are neighbors along a diagonal as shown in Fig. 13. For example, $\mathcal{G}_{m, m}$ or $\mathcal{H}_{m, m}$ may represent a shopping complex, with the nodes corresponding to the locations of shops with WiFi Access Points (AP) for Internet access. Depending on the proximity of the shops to each other and the transmission ranges of the APs, the conflict graph could be $\mathcal{G}_{m, m}$ or $\mathcal{H}_{m, m} . \mathcal{H}_{m, m}$ is also the conflict graph of a cellular network with square cells as shown in Fig. 14.

3) Let $\mathcal{T}_{m, m, m}$ be a three-dimensional grid graph (see Fig. 6), which may, for example, be the conflict graph for offices in a corporate building or rooms in a hotel.

4) The conflict graph (Fig. 8) of a cellular network with hexagonal cells (Fig. 7).

5) Consider a clique ${ }^{5}$ of size $e$, where $e \geq 1$ is any integer. This is the conflict graph for any set of $e$ locations that are close to each other.

All of the above are mean valid graphs:

Theorem 1: The following graphs are mean valid, with $d$, the number of disjoint maximal I.S., indicated in each case:

1) a clique of size $e \geq 1(d=e)$,

2) a line graph $\mathcal{G}_{m}(d=2)$,

3) a two-dimensional grid graph $\mathcal{G}_{m, m}(d=2)$,

4) a two-dimensional grid graph $\mathcal{H}_{m, m}(d=4)$,

5) a three-dimensional grid graph $\mathcal{T}_{m, m, m}(d=8)$.

6) a cellular network with hexagonal cells, under Assumption 1 in Section VI-A $(d=3)$.

We defer the proof of Theorem 1 to Section VI-A. Also, as we show in Section VI-B, some other common classes of graphs, such as a star and a $\kappa$-regular bipartite graph, are mean valid as well.

A graph obtained by considering the union of disjoint mean valid graphs, all of which correspond to the same integer $d$, and then adding some edges to get a connected graph, is a mean valid graph under some technical conditions ${ }^{6}$, e.g., the cellular networks in a group of neighboring towns or the $\mathrm{WiFi}$ networks in the departments of a university campus. Fig. 1 illustrates the latter example.

\footnotetext{
${ }^{5}$ Recall that a clique or a complete graph of size $e$ is a graph with $e$ nodes and an edge between every pair of nodes [4].

${ }^{6}$ These technical conditions are stated in Lemmas 10 and 11 in Section VI-A.
} 


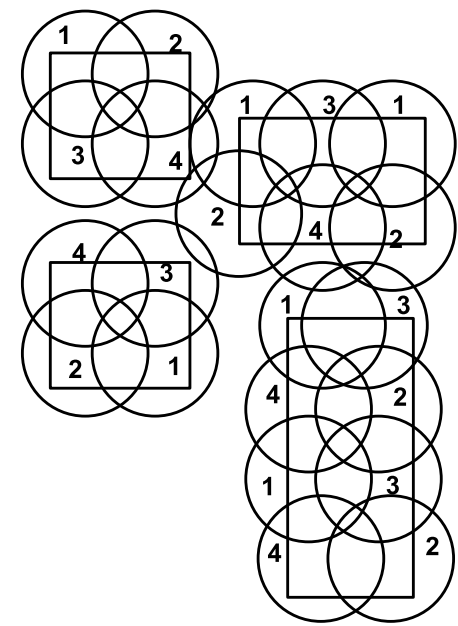

Fig. 1. The rectangles represent departments in a university campus and the circles are the ranges of WiFi access points. The circles (nodes) in each rectangle constitute a grid graph $\mathcal{H}_{m, m}$, which is mean valid with $d=4$ (see part 4 of Theorem 1). The overall graph is also mean valid with $d=4$. With $I_{j}, j \in\{1,2,3,4\}$, being disjoint maximal I.S. as in Definition 3, the number in each circle indicates the I.S. to which the corresponding node belongs, i.e. nodes corresponding to the circles numbered $j \in\{1,2,3,4\}$ belong to I.S. $I_{j}$.

\section{A Necessary and Sufficient Condition}

We state a property of mean valid graphs for later use.

Lemma 3: Let $G=(V, E)$ be a graph that satisfies Condition 1 in Definition 3. Suppose $I \in \mathscr{I}$ contains $m_{j}(I)$ nodes from $I_{j}, j=1, \ldots, d$. $G$ is mean valid if and only if:

$$
\sum_{j=1}^{d} \frac{m_{j}(I)}{M_{j}} \leq 1 \quad \forall I \in \mathscr{I}
$$

\section{SyMmetriC NE IN MEAN VALID GRAPHS}

In this section, we show that a mean valid graph has a unique symmetric $\mathrm{NE}$; in this $\mathrm{NE}$, in the notation of Definition 3, primaries offer bandwidth at all the nodes in $I_{j}$, $j \in\{1, \ldots, d\}$, with the same probability $t_{j}$, i.e. $\alpha_{j, l}=t_{j}$ $\forall l=1, \ldots, M_{j}$, where $\left\{t_{j}: j=1, \ldots, d\right\}$ is the unique solution of a set of equations that we provide.

First, we will argue, by contradiction, that for each $j$, $\alpha_{j, l}=\bar{\alpha}_{j} \forall l=1, \ldots, M_{j}$, where $\bar{\alpha}_{j}$ is given by (11). In the symmetric NE $(\psi, \ldots, \psi)$, by (7) and the discussion just after it, primary 1 gets an expected payoff of $W\left(\alpha_{j, l}\right)$ at node $a_{j, l}$; also, by (8), its total expected payoff is:

$$
E\left\{u_{1}\left(\psi, \psi_{-1}\right)\right\}=\sum_{j=1}^{d} \sum_{l=1}^{M_{j}} \alpha_{j, l} W\left(\alpha_{j, l}\right)
$$

Suppose $\alpha_{j, l}, l=1, \ldots, M_{j}$ are not all equal for some $j$. By (7) and Lemma $1, W(\alpha)$ is a strictly decreasing function of $\alpha$; so primary 1 offers bandwidth with a high probability $\alpha_{j, l}$ at nodes $a_{j, l}$ at which it gets a low payoff $W\left(\alpha_{j, l}\right)$. This seems to suggest that primary 1 could get a higher overall payoff by unilaterally switching to an alternative strategy, say $\psi_{0}$, under which it decreases (respectively, increases) the node probabilities at nodes that yield a low (respectively, high) payoff, if such a strategy $\psi_{0}$ were to exist. This would contradict the fact that the distribution $\left\{\alpha_{j, l}: j=1, \ldots, d ; l=1, \ldots, M_{j}\right\}$ is primary 1 's best response and thereby imply that $\alpha_{j, l}, l=1, \ldots, M_{j}$ must be equal for every $j=1, \ldots, d$.

The existence of such a strategy $\psi_{0}$ is guaranteed by Condition 2 in Definition 3- that (10) holds for every valid distribution. Let $\psi_{0}$ be a strategy under which primary 1 offers bandwidth at each node in $I_{j}, j \in\{1, \ldots, d\}$ w.p. $\bar{\alpha}_{j}$. Note that $\sum_{j=1}^{d} \bar{\alpha}_{j} \leq 1$ by $(10)$; so $\psi_{0}$ is a valid distribution since it corresponds to the I.S. distribution $\beta\left(I_{j}\right)=\bar{\alpha}_{j}$, $j \in\{1, \ldots, d\}, \beta\left(I_{\emptyset}\right)=1-\sum_{j=1}^{d} \bar{\alpha}_{j}, \beta(I)=0, I \neq$ $I_{1}, \ldots, I_{d}, I_{\emptyset}$. By $(8)$, the total expected payoff of primary 1 if it plays strategy $\psi_{0}$ is:

$$
E\left\{u_{1}\left(\psi_{0}, \psi_{-1}\right)\right\}=\sum_{j=1}^{d} \sum_{l=1}^{M_{j}} \bar{\alpha}_{j} W\left(\alpha_{j, l}\right)
$$

By (13) and (14):

$$
\begin{aligned}
& E\left\{u_{1}\left(\psi, \psi_{-1}\right)\right\}-E\left\{u_{1}\left(\psi_{0}, \psi_{-1}\right)\right\} \\
= & \sum_{j=1}^{d}\left(\sum_{l=1}^{M_{j}} \alpha_{j, l} W\left(\alpha_{j, l}\right)-\bar{\alpha}_{j}\left(\sum_{l=1}^{M_{j}} W\left(\alpha_{j, l}\right)\right)\right)
\end{aligned}
$$

Now, we have the following algebraic fact, proved in the Appendix.

Lemma 4: Let $N \geq 2$ be an integer, $\alpha_{1}, \ldots, \alpha_{N}$ be real numbers and $\bar{\alpha}=\frac{\sum_{i=1}^{N} \alpha_{i}}{N}$. Let $f(x)$ be any strictly decreasing function of $x$. Then:

$$
\left(\sum_{i=1}^{N} \alpha_{i} f\left(\alpha_{i}\right)\right) \leq \bar{\alpha}\left(\sum_{i=1}^{N} f\left(\alpha_{i}\right)\right)
$$

with equality iff $\alpha_{1}=\ldots=\alpha_{N}=\bar{\alpha}$.

Intuitively, since $f($.$) is strictly decreasing, in the LHS of$ (16), the terms in which $f\left(\alpha_{i}\right)$ is large are multiplied by small factors $\alpha_{i}$ and vice-versa; on the other hand, all terms $f\left(\alpha_{i}\right)$ on the RHS are multiplied by the same factor $\bar{\alpha}$. So the LHS is smaller.

Now, as mentioned above, $f(\alpha)=W(\alpha)=1-w(q \alpha, n)$ is a strictly decreasing function of $\alpha$. So by Lemma 4, the expression in (15) is $\leq 0$, with equality holding iff $\alpha_{j, 1}=$ $\ldots=\alpha_{j, M_{j}}=\bar{\alpha}_{j}$ for each $j \in\{1, \ldots, d\}$. But since $\psi$ is a best response, $E\left\{u_{1}\left(\psi, \psi_{-1}\right)\right\} \geq E\left\{u_{1}\left(\psi_{0}, \psi_{-1}\right)\right\}$. So the expression in (15) must equal 0 and hence $\alpha_{j, 1}=\ldots=$ $\alpha_{j, M_{j}}=\bar{\alpha}_{j}$ for each $j \in\{1, \ldots, d\}$.

Now, suppose $\sum_{j=1}^{d} \bar{\alpha}_{j}<1$. Then primary 1 can unilaterally offer bandwidth at each node in $I_{d}$ with probability $1-\sum_{j=1}^{d-1} \bar{\alpha}_{j}>\bar{\alpha}_{d}$ instead of $\bar{\alpha}_{d}$ and increase its payoff. This contradicts the fact that the distribution is a NE. So we must have $\sum_{j=1}^{d} \bar{\alpha}_{j}=1$. Thus, we have shown the following:

Lemma 5: In a mean valid graph, under every symmetric NE, each primary offers bandwidth at each node in $I_{j}$ w.p. $t_{j}, j \in\{1, \ldots, d\}$, for some $t_{j} \geq 0, j=1, \ldots, d$, where $\sum_{j=1}^{d} t_{j}=1$.

A typical way in which the node probability distribution $\alpha_{j, l}=$ $t_{j} \forall l=1, \ldots, M_{j}$, arises is via the I.S. distribution $\beta\left(I_{j}\right)=$ $t_{j}, j=1, \ldots, d ; \beta(I)=0 \forall I \neq I_{1}, \ldots, I_{d}$.

The following result provides necessary conditions for a distribution $\left\{t_{j}: j=1, \ldots, d\right\}$ as in Lemma 5 to constitute a symmetric NE. 
Lemma 6: If a distribution $\left\{t_{j}: j=1, \ldots, d\right\}$ as in Lemma 5 constitutes a symmetric NE, then $I_{1}, \ldots, I_{d^{\prime}}$ are best responses and $I_{d^{\prime}+1}, \ldots, I_{d}$ are not, for some integer $d^{\prime} \in\{1, \ldots, d\}$. Also, each $I \in \mathscr{I}$ containing a node from $I_{j}$ for some $j>d^{\prime}$ is not a best response. Hence:

$$
t_{j}=0, j>d^{\prime} .
$$

Intuitively, a primary prefers to offer bandwidth at a large I.S. because it gets some revenue at every node in the I.S. it selects and its total payoff is the sum of the revenues at the nodes of the I.S. Also, recall that by (9), $I_{1}, \ldots, I_{d}$ are in decreasing order of size. So a primary will (i) try to offer bandwidth only at the largest I.S. $I_{1}$, (ii) offer bandwidth at the next largest I.S. $I_{2}$ as well with some probability only if the competition at $I_{1}$ increases beyond a threshold, (iii) offer bandwidth at $I_{3}$ as well with some probability only if the competition at $I_{1}$ and $I_{2}$ increases beyond a certain threshold and so on. Hence, the set of best responses out of $I_{1}, \ldots, I_{d}$ is of the form $I_{1}, I_{2}, \ldots, I_{d^{\prime}}$ for some $1 \leq d^{\prime} \leq d$.

Now, if primary $i$ offers bandwidth at I.S. $I^{\prime} \in \mathscr{I}$, its overall expected payoff, denoted by $U_{1}\left(I^{\prime}\right)$, is the sum of the expected payoffs at the nodes in $I^{\prime}$, which, by (7) and the discussion just after it, is given by:

$$
U_{1}\left(I^{\prime}\right)=\sum_{v \in I^{\prime}} W\left(\alpha_{v}\right)=\sum_{v \in I^{\prime}}\left(1-w\left(q \alpha_{v}, n\right)\right) .
$$

Now, consider a symmetric NE with $\left\{t_{j}: j=1, \ldots, d\right\}$ as in Lemma 5. By (18) and the fact that $\left|I_{j}\right|=M_{j}$, the payoff of primary 1 if it offers bandwidth at $I_{j}$ is:

$$
U_{1}\left(I_{j}\right)=M_{j} W\left(t_{j}\right)
$$

By Lemma $6, I_{1}, \ldots, I_{d^{\prime}}$ are best responses and $I_{d^{\prime}+1}$ is not. So:

$$
U_{1}\left(I_{1}\right)=\ldots=U_{1}\left(I_{d^{\prime}}\right)>U_{1}\left(I_{d^{\prime}+1}\right)
$$

Substituting (19) into the above and using (17) and the fact that $W(0)=1-w(0, n)=1$, we get:

$$
M_{1} W\left(t_{1}\right)=\ldots=M_{d^{\prime}} W\left(t_{d^{\prime}}\right)>M_{d^{\prime}+1}
$$

Thus, we have shown the following:

Lemma 7: A distribution $\left\{t_{j}: j=1, \ldots, d\right\}$ as in Lemma 5 that constitutes a symmetric NE must satisfy (17) and (20) for some integer $d^{\prime} \in\{1, \ldots, d\}$.

Lemma 7 provides necessary conditions for a distribution $\left\{t_{j}: j=1, \ldots, d\right\}$ to constitute a symmetric NE. The following lemma shows that these conditions are sufficient as well.

Lemma 8: Let $1 \leq d^{\prime} \leq d$ and $t_{1}, \ldots, t_{d}$ be a probability distribution such that (17) and (20) hold. Then the symmetric strategy profile in which every primary offers bandwidth at each node in $I_{j}$ w.p. $t_{j}, j \in\{1, \ldots, d\}$, is a NE.

The proof of Lemma 8 (see the Appendix) is based on the fact that the graph, being mean valid, satisfies Condition 2 in Definition 3.

The following technical lemma shows the existence and uniqueness of a distribution $\left(t_{1}, \ldots, t_{d}\right)$ satisfying (17) and (20) for every value of $q$.
Lemma 9: For every $q \in(0,1)$, there exists a unique integer $d^{\prime}=d^{\prime}(q)$ and a unique probability distribution $\left(t_{1}, \ldots, t_{d}\right)$ such that (17) and (20) hold. Also, $d^{\prime}(q)$ is an increasing function of $q$ and, for every value of $q, t_{1} \geq t_{2} \ldots \geq t_{d}$.

Note that the fact that $d^{\prime}(q)$ is an increasing function of $q$ is consistent with the intuition that for small values of $q$, primaries tend to offer bandwidth at only the larger I.S. out of $I_{1}, \ldots, I_{d}$, and as $q$, and thereby the competition from other primaries increases, they also choose the smaller ones. Also, the fact that $t_{1} \geq t_{2} \ldots \geq t_{d}$ for all $q$ is consistent with the intuition that primaries offer bandwidth at the larger I.S. with a larger probability.

Finally, putting together the above discussion, we get the main result of this section:

Theorem 2: In a mean valid graph, for every $q \in(0,1)$, there is a unique symmetric NE; in this NE, each primary offers bandwidth at every node in $I_{j}, j \in\{1, \ldots, d\}$, w.p. $t_{j}$, i.e. $\alpha_{j, l}=t_{j}, l=1, \ldots, M_{j}$, where $\left(t_{1}, \ldots, t_{d}\right)$ is the unique distribution satisfying (17) and (20).

Proof: By Lemma 5, under every symmetric NE, each primary must offer bandwidth at all the nodes in $I_{j}$, $j \in\{1, \ldots, d\}$, w.p. $t_{j}$ for some probability distribution $\left(t_{1}, \ldots, t_{d}\right)$. Also, by Lemma $7,(17)$ and (20) hold for this distribution. By Lemma 9, for a fixed value of $q \in(0,1)$, there exists a unique distribution $\left(t_{1}, \ldots, t_{d}\right)$ satisfying (17) and (20). Finally, by Lemma 8, the strategy profile where each primary uses this distribution is a NE. The result follows.

Thus, every mean valid graph has a unique symmetric NE, which can be explicitly computed by solving the system of equations (17) and (20).

Example: Suppose there are $n=2$ primaries and $k=1$ secondary. Consider a grid graph $\mathcal{H}_{m, m}$, which was introduced in Section IV-B, with $m=7$ (see Fig. 13). By part 4 of Theorem 1, this is a mean valid graph and, in the notation of Definition 3, $d=4$, the I.S. $I_{1}, I_{2}, I_{3}$ and $I_{4}$ are as described in Section VI-A, and $M_{1}=16, M_{2}=M_{3}=12, M_{4}=9$. The symmetric NE is of the form described in Theorem 2 with $d^{\prime}(q), t_{1}, t_{2}, t_{3}$ and $t_{4}$ for different $q \in(0,1)$ as follows:

1) For $0<q<\frac{1}{4}, d^{\prime}=1, t_{1}=1, t_{2}=t_{3}=t_{4}=0$.

2) For $\frac{1}{4} \leq q<\frac{15}{16}, d^{\prime}=3, t_{1}=\frac{1}{11}\left(3+\frac{2}{q}\right), t_{2}=t_{3}=$ $\frac{1}{11}\left(4-\frac{1}{q}\right) t_{4}=0$.

3) For $\frac{15}{16} \leq q<1, d^{\prime}=4, t_{1}=\frac{1}{49}\left(9+\frac{13}{q}\right), t_{2}=t_{3}=$ $\frac{1}{49}\left(\frac{1}{q}+12\right) t_{4}=\frac{1}{49}\left(16-\frac{15}{q}\right)$.

Note that, consistent with Theorem $2, d^{\prime}(q)$ is an increasing function of $q$ and $t_{1} \geq t_{2} \geq t_{3} \geq t_{4}$ for each value of $q$. In fact, for all $q, t_{2}=t_{3}$, which is because $I_{2}$ and $I_{3}$ are of the same size. Fig. 2 plots $t_{1}, t_{2}$ and $t_{4}$ versus $q$. For small $q$, primaries offer bandwidth at the largest I.S. $I_{1}$ with probability 1 ; but as $q$ increases, the competition at $I_{1}$ increases, inducing the primaries to shift probability mass from $I_{1}$ to the other I.S. So $t_{1}$ decreases in $q$. However, note that for all values of $q, t_{1} \geq t_{2} \geq t_{4}$ and $t_{4}$ is very small (less than 0.02 ).

Remark 1: The unique symmetric NE need not be pure even with respect to the node selections, as the above example shows. However, this mixed choice is not really an artifact of mixed price choice. For instance, consider a scenario where 


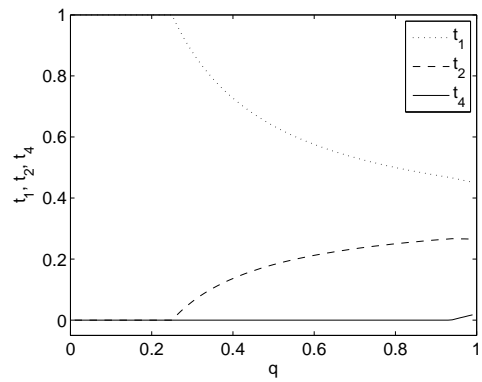

Fig. 2. The figure shows the symmetric NE probabilities $t_{1}, t_{2}$ and $t_{4}$ for the example after Theorem 2.

all primaries must choose the same fixed price $p_{0}$ (perhaps the prices have been standardized because of government regulation). Suppose there are two nodes $v_{1}$ and $v_{2}$ connected by an edge, two primaries $(n=2)$ and one secondary at each node $(k=1)$. Then it is easy to show that the strategy profile under which each primary offers bandwidth at $v_{1}$ and $v_{2}$ w.p. $1 / 2$ each constitutes the unique symmetric NE.

The intuition behind randomization across different I.S. in a symmetric NE is that primaries would like to offer bandwidth at an I.S. at which other primaries do not offer bandwidth with a high probability, whereas in a symmetric NE that is pure with respect to the node selection, all primaries offer bandwidth at the same I.S.

\section{Some Specific Mean VAlid Graphs}

Theorem 2 provides the form of the symmetric NE in mean valid graphs. So in this section, we identify some classes of mean valid graphs.

\section{A. Topologies that Commonly Arise in Practice}

We now prove Theorem 1.

The proof of part 1 of Theorem 1 is straightforward: let $\left\{v_{1}, \ldots, v_{e}\right\}$ be the nodes of the clique. $I_{j}=\left\{v_{j}\right\}, j=$ $1, \ldots, e$ are disjoint maximal I.S. whose union is $V$. Also, these are the only I.S. in the graph; so (12) holds and the clique is mean valid by Lemma 3 .

Next, we prove some lemmas that we use to prove the other parts of Theorem 1 .

Lemma 10: Let $G=(V, E)$ be a mean valid graph, where $V=I_{1} \cup \ldots \cup I_{d}$ and $I_{1}, \ldots, I_{d}$ are disjoint maximal I.S. Let $E^{\prime} \supseteq E$ be any set such that no edge in $E^{\prime}$ is between two nodes in the same I.S. $I_{j}, j \in\{1, \ldots, d\}$. Then the graph $G^{\prime}=\left(V, E^{\prime}\right)$ is mean valid.

Thus, if a graph $G$ is mean valid, then the graph $G^{\prime}$ obtained by adding edges in any fashion to $G$, while ensuring that $I_{j}$, $j=1, \ldots, d$ continue to be I.S. in $G^{\prime}$, is a mean valid graph as well.

Lemma 11: Suppose for each $i=1, \ldots, N, G^{i}=\left(V^{i}, E^{i}\right)$ is a mean valid graph, where $V^{i}=I_{1}^{i} \cup \ldots \cup I_{d}^{i}, I_{1}^{i}, \ldots, I_{d}^{i}$ are disjoint maximal I.S., and $\left|I_{j}^{i}\right|=M_{j}^{i}, j=1, \ldots, d$. Let $\mathbf{M}^{i}=\left(M_{1}^{i}, \ldots, M_{d}^{i}\right)$. If

$$
\mathbf{M}^{i}=c_{i} \mathbf{M}^{0}, \quad i=1, \ldots, N
$$

for some vector $\mathbf{M}^{0}=\left(M_{1}^{0}, \ldots, M_{d}^{0}\right)$ and positive scalars $c_{1}, \ldots, c_{N}$, then $G=\left(\cup_{i=1}^{N} V^{i}, \cup_{i=1}^{N} E^{i}\right)$ is mean valid.

Lemma 11 says that if $G^{i}, i=1, \ldots, N$ are mean valid graphs, then their union $G$ is a mean valid graph as well provided each of $G^{i}, i=1, \ldots, N$ contains (i) the same number, $d$, of disjoint maximal I.S., and (ii) the same proportion of nodes in the $d$ I.S. $I_{1}^{i}, \ldots, I_{d}^{i}$. Since the union graph $G$ is a disconnected graph with $N$ components, Lemma 11 is not useful by itself to prove that a graph is mean valid. But it can be effectively used in conjunction with Lemma 10 to combine a set of $N$ mean valid graphs into a new connected mean valid graph by (i) first considering their union, which is a disconnected graph, (ii) and then adding some edges to it to make it connected.

A useful special case is when each of these $N$ graphs $G^{i}$ is a clique of size $d$ (which is mean valid by Part 1 of Theorem 1) with vertex set $V^{i}=\left\{v_{1}^{i}, \ldots, v_{d}^{i}\right\}$. Note that these graphs satisfy the hypothesis of Lemma 11 with $I_{j}^{i}=\left\{v_{j}^{i}\right\}, M_{j}^{i}=1$, $\forall i, j, \mathbf{M}^{0}=(1, \ldots, 1)$ and $c_{i}=1 \forall i$. This special case can be used to prove the mean validity of several of the graphs mentioned in Theorem 1, as we explain below.

For an integer $m \geq 1$, let $m_{e}$ (respectively, $m_{o}$ ) denote the greatest even (respectively, odd) integer less than or equal to $m$.

We now prove part 2 of Theorem 1. Consider a linear graph $\mathcal{G}_{m}$ with node set $\left\{v_{1}, v_{2}, \ldots, v_{m}\right\}$ as shown in part (a) of Fig. 3. First, let $m$ be even- say $m=2 N$. For $i=1, \ldots, N$, let $G^{i}$ be the clique of size 2 with the node set $V^{i}=\left\{v_{2 i-1}, v_{2 i}\right\}$ and the edge between the two nodes. In the notation of Lemma 11, let $I_{1}^{i}=\left\{v_{2 i-1}\right\}$ and $I_{2}^{i}=\left\{v_{2 i}\right\}$. By Lemma $11, G=G^{1} \cup G^{2} \cup \ldots \cup G^{N}$ is a mean valid graph with $d=2$ and the disjoint maximal I.S. $I_{1}=\left\{v_{1}, v_{3}, v_{5}, \ldots v_{m_{o}}\right\}$ and $I_{2}=\left\{v_{2}, v_{4}, v_{6}, \ldots, v_{m_{e}}\right\}$. We can obtain $\mathcal{G}_{m}$ by adding the edges $\left(v_{2}, v_{3}\right),\left(v_{4}, v_{5}\right), \ldots,\left(v_{2 N-2}, v_{2 N-1}\right)$ to $G$ as illustrated in part (a) of Fig. 4. Note that no edge is between two nodes in the same I.S. $I_{j}, j \in\{1,2\}$; so the hypothesis of Lemma 10 is satisfied. Hence, $\mathcal{G}_{m}$ is mean valid by Lemma 10 . The proof of the fact that $\mathcal{G}_{m}$ is also mean valid for $m$ odd is deferred to the Appendix.

Now, we prove part 3 of Theorem 1. Consider $\mathcal{G}_{m, m}$, where $m$ may be odd or even. Let $v_{i j}$ be the node in the $i$ 'th row and $j$ 'th column $i, j \in\{1, \ldots, m\}$ (see part (b) of Fig. 3). We start with a line graph $\mathcal{G}_{m^{2}}$, which is mean valid by part 2 of Theorem 1, and add some edges to obtain $\mathcal{G}_{m, m}$ as shown in Fig. 5. Specifically, let $\mathcal{G}_{m^{2}}$ be the line graph with the set of nodes $\left\{v_{1,1}, v_{1,2}, \ldots, v_{1, m}, v_{2, m}, v_{2, m-1}, \ldots, v_{2,1}, v_{3,1}\right.$, $\left.v_{3,2}, \ldots, v_{3, m}, v_{4, m}, v_{4, m-1}, \ldots\right\}$ and an edge between each pair of consecutive nodes in this order. $\mathcal{G}_{m^{2}}$ is mean valid with $d=2$, and the disjoint maximal I.S. $I_{1}=\left\{v_{11}, v_{13}\right.$, $\left.\ldots, v_{1, m_{o}}, v_{22}, v_{24}, \ldots, v_{2, m_{e}}, v_{31}, v_{33}, \ldots, v_{3, m_{o}}, \ldots\right\}$ and $I_{2}=\left\{v_{12}, v_{14}, \ldots, v_{1, m_{e}}, v_{21}, v_{23}, \ldots, v_{2, m_{o}}, v_{32}, v_{34}, \ldots\right.$, $\left.v_{3, m_{e}}, \ldots\right\} . \mathcal{G}_{m, m}$ can be obtained from $\mathcal{G}_{m^{2}}$ by adding the remaining edges shown dotted in Fig. 5. Note that no edge is between the same I.S. $I_{j}, j=1,2$. So $\mathcal{G}_{m, m}$ is mean valid by Lemma 10.

Next, we prove part 4 of Theorem 1. Consider $\mathcal{H}_{m, m}$ (see Fig. 13). As in $\mathcal{G}_{m, m}$, let $v_{i j}$ be the node in the $i$ 'th row and $j$ 'th column. Let $d=4, I_{1}=\left\{v_{11}, v_{13}, v_{15}, \ldots, v_{1, m_{o}}, v_{31}\right.$, 


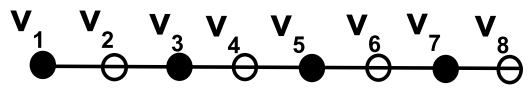

(a)

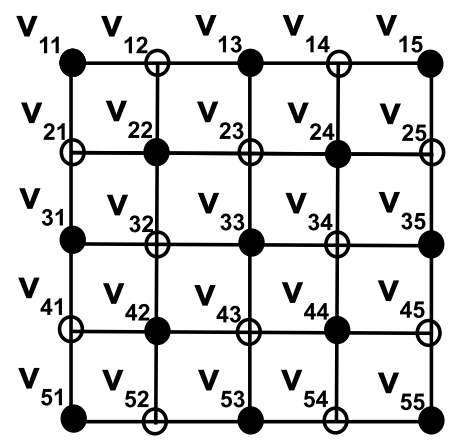

(b)

Fig. 3. Part (a) shows a linear graph, $\mathcal{G}_{m}$, with $m=8$ and part (b) shows a grid graph, $\mathcal{G}_{m, m}$, with $m=5$. In both graphs, the darkened and un-darkened nodes constitute $I_{1}$ and $I_{2}$ respectively.

$\left.v_{33}, v_{35}, \ldots, v_{3, m_{o}}, \ldots\right\}, I_{2}=\left\{v_{12}, v_{14}, v_{16}, \ldots, v_{1, m_{e}}\right.$, $\left.v_{32}, v_{34}, v_{36}, \ldots, v_{3, m_{e}}, \ldots\right\}, I_{3}=\left\{v_{21}, v_{23}, v_{25}, \ldots, v_{2, m_{o}}\right.$, $\left.v_{41}, v_{43}, v_{45}, \ldots, v_{4, m_{o}}, \ldots\right\}$ and $I_{4}=\left\{v_{22}, v_{24}, v_{26}, \ldots\right.$, $\left.v_{2, m_{e}}, v_{42}, v_{44}, v_{46}, \ldots, v_{4, m_{e}}, \ldots\right\}$ (see part (b) of Fig. 4). Note that $I_{1}, I_{2}, I_{3}$ and $I_{4}$ are disjoint maximal I.S. For $i, j \in$ $\{1, \ldots, m-1\}$, let $C_{i, j}$ be the clique consisting of the nodes $\left\{v_{i, j}, v_{i, j+1}, v_{i+1, j}, v_{i+1, j+1}\right\}$ and the edges among them (see Fig. 15). First, let $m$ be even. The proof that $\mathcal{H}_{m, m}$ is mean valid is similar to the above proof of mean validity of $\mathcal{G}_{m}$ with $m$ even: we can obtain $\mathcal{H}_{m, m}$ by considering the union of the cliques $C_{i, j}, i, j \in\{1,3,5, \ldots, m-1\}$, which is a mean valid graph by Lemma 11, and then adding the remaining edges as illustrated in part (b) of Fig. 4. Note that no edge is between two nodes in the same I.S. $I_{j}, j \in\{1,2,3,4\}$; so the hypothesis of Lemma 10 is satisfied. Hence, $\mathcal{H}_{m, m}$ is mean valid by Lemma 10 . The proof of the fact that $\mathcal{H}_{m, m}$ is also mean valid for $m$ odd is deferred to the Appendix.

The proof of part 5 of Theorem 1 is similar to that of part 4: we outline the differences. For $i, j, l \in\{1, \ldots, m\}$, let $v_{i j l}$ be the node in the $i$ 'th row, $j$ 'th column and $l$ 'th level (in the direction normal to the plane of the paper). The node set of $\mathcal{T}_{m, m, m}$ can be partitioned into 8 disjoint maximal I.S. $I_{1}, \ldots, I_{8}$ similar to $I_{1}, \ldots, I_{4}$ for $\mathcal{H}_{m, m}$ (see Fig. 6). Also, cliques $C_{i j l}, i, j, l \in\{1, \ldots, m-1\}$ of size 8 each can be defined similar to the cliques $C_{i j}$ for $\mathcal{H}_{m, m}$. For $m$ even, we can obtain $\mathcal{T}_{m, m, m}$ by considering the union of the cliques $C_{i j l}, i, j, l \in\{1,3,5, \ldots, m-1\}$ and then adding the remaining edges. The fact that $\mathcal{T}_{m, m, m}$ is mean valid then follows from Lemmas 11 and 10. The proof of the fact that $\mathcal{T}_{m, m, m}$ is also mean valid for $m$ odd is outlined in the Appendix.

We now prove part 6 of Theorem 1. Consider a cellular network as shown in Fig. 7, whose conflict graph is shown in Fig. 8. The nodes in the graph can be partitioned into three disjoint maximal I.S. $I_{1}, I_{2}$ and $I_{3}$ as shown in Fig. 8. We consider this conflict graph with the following assumption,

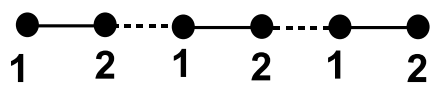

(a)

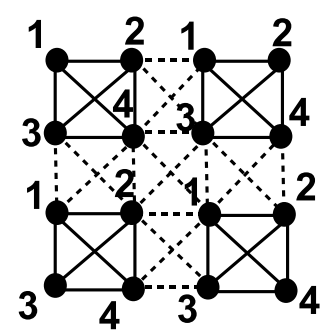

(b)

Fig. 4. Part (a) (respectively, part (b)) shows the construction of $\mathcal{G}_{6}$ (respectively, $\mathcal{H}_{4,4}$ ) from 3 (respectively, 4) cliques of size 2 (respectively, 4) each. The solid edges constitute the cliques $G^{1}, G^{2}, G^{3}$ (respectively, $C_{1,1}, C_{1,3}, C_{3,1}$ and $C_{3,3}$ ) and the dotted edges are those that are added later. The numbers next to the nodes shows the I.S. they are in, i.e., a node labeled $j$ is in I.S. $I_{j}$, where $j \in\{1,2\}$ (respectively, $j \in\{1,2,3,4\}$ ). Note that no edge is between two nodes in the same I.S. $I_{j}$; so the hypothesis of Lemma 10 is satisfied.

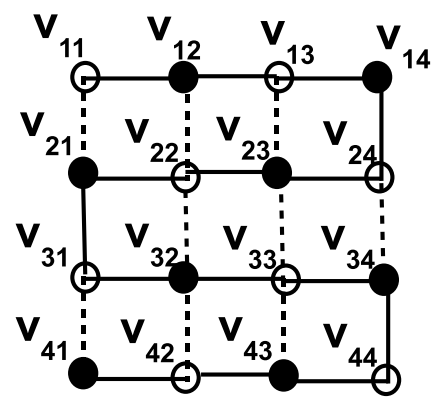

Fig. 5. The figure shows the construction of the grid graph $\mathcal{G}_{m, m}$ from the line graph $\mathcal{G}_{m^{2}}$ for $m=4$. The solid edges constitute $\mathcal{G}_{m^{2}}$ and the dotted edges are later added to obtain $\mathcal{G}_{m}, m$. The un-darkened and darkened nodes constitute $I_{1}$ and $I_{2}$ respectively in both $\mathcal{G}_{m^{2}}$ and $\mathcal{G}_{m, m}$. Note that no edge is between a node in $I_{1}$ and a node in $I_{2}$, so the hypothesis of Lemma 10 is satisfied.

which eliminates problems arising due to boundary effects.

Assumption 1: There are an even number, say $r$, of rows of nodes, each containing $3 \eta$ nodes, for some integer $\eta \geq 1$.

Under this assumption, as illustrated in Fig. 8, the graph can be obtained by considering the union of $r \eta$ disjoint cliques of size 3 each, which is a mean valid graph by Lemma 11, and then adding some edges. Note that no edge is between two nodes in the same I.S. $I_{j}, j \in\{1,2,3\}$ (see Fig. 8); so the hypothesis of Lemma 10 is satisfied. Hence, the graph is mean valid by Lemma 10 .

Note that the above proof goes through if the graph can be partitioned into cliques of size 3 even if Assumption 1 is not satisfied. If the graph cannot be partitioned into cliques of size 3 , then the analysis is more complicated because of boundary effects. We omit this analysis for brevity.

\section{B. Some Other Classes of Mean Valid Graphs}

In this subsection, we show that some other common classes of graphs are mean valid. We focus on connected bipartite graphs [4], which are of the form $G=(V, E)$ where $V=$ 


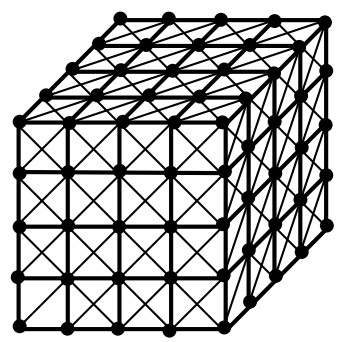

(a)

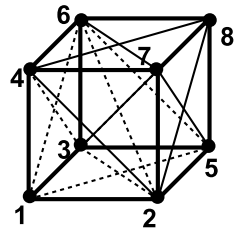

(b)
Fig. 6. Part (a) shows a three-dimensional grid graph $\mathcal{T}_{m, m, m}$ for $m=5$. It consists of periodic repetitions of the graph shown in part (b). Also, in part (b), the node labels show the I.S. $I_{1}, \ldots, I_{8}$ they are in, i.e. a node with the label $j$ is part of the I.S. $I_{j}, j \in\{1, \ldots, 8\}$.

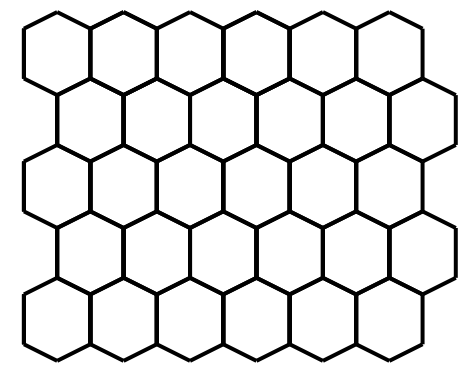

Fig. 7. The figure shows a tiling of a plane with hexagons, e.g. cells in a cellular network. Transmissions at neighboring cells interfere with each other.

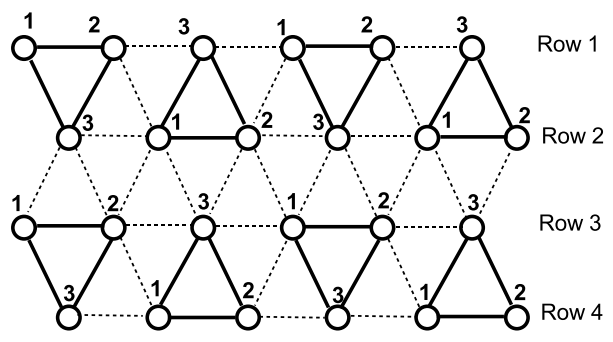

Fig. 8. The figure shows the conflict graph of a hexagonal tiling of a plane. Both the solid and dotted edges are part of the graph. The nodes labelled $j$, $j \in\{1,2,3\}$, are in I.S. $I_{j}$. There are four rows of nodes. The figure also shows the construction of the graph from cliques of size 3 each, shown by the solid edges. The dotted edges are added later. Note that no edge is between two nodes in the same I.S., so the hypothesis of Lemma 10 is satisfied.

$A \cup B$ and every edge is between a node in $A$ and a node in $B$. Without loss of generality, suppose $|A| \leq|B|$. In the notation of Definition 3, $d=2, I_{1}=B$ and $I_{2}=A$. Also, a necessary condition for a node distribution $\left\{\alpha_{i}, i \in A ; \gamma_{j}, j \in B\right\}$, under which bandwidth is offered at node $i \in A$ (respectively, $j \in B$ ) w.p. $\alpha_{i}$ (respectively, $\gamma_{j}$ ), to be valid is that

$$
\alpha_{i}+\gamma_{j} \leq 1 \forall(i, j) \in E .
$$

This is because, if $\alpha_{i}+\gamma_{j}>1$ for some $(i, j) \in E$, then with a positive probability bandwidth would be offered at both nodes $i$ and $j$, which are neighbors.

Recall that a $\kappa$-regular graph is one in which the degree of every node is $\kappa$ [4].

Proposition 1: A $\kappa$-regular bipartite graph is mean valid.

Proof: Let $|A|=N$ and $|B|=M$. First, we show that $N=M$. Since $\kappa$ edges are incident upon each node in $A$,
$|E|=|A| \kappa=N \kappa$. Similarly, $|E|=M \kappa$. So $N=M$.

Now, let $\left\{\alpha_{i}, i \in A ; \gamma_{j}, j \in B\right\}$ be a valid distribution. Adding (22) over all $(i, j) \in E$, we get:

$$
\sum_{(i, j) \in E}\left(\alpha_{i}+\gamma_{j}\right) \leq|E|=N \kappa
$$

But since exactly $\kappa$ edges are incident on each node:

$$
\sum_{(i, j) \in E}\left(\alpha_{i}+\gamma_{j}\right)=\kappa\left(\sum_{i \in A} \alpha_{i}+\sum_{j \in B} \gamma_{j}\right)
$$

By (23) and (24),

$$
\frac{\sum_{i \in A} \alpha_{i}}{N}+\frac{\sum_{j \in B} \gamma_{j}}{N} \leq 1
$$

So Condition 2 in Definition 3 is satisfied and the graph is mean valid.

Recall that a star is a graph with a node $a_{1}$ called the center, nodes $b_{1}, \ldots, b_{M}$ called the leaves, and edges $\left(a_{1}, b_{j}\right), j=$ $1, \ldots, M$ [4]. Note that this is a bipartite graph with edges only between the sets $A=\left\{a_{1}\right\}$ and $B=\left\{b_{1}, \ldots, b_{M}\right\}$.

Proposition 2: A star is mean valid. (22),

Proof: Let $\left\{\alpha_{1}, \gamma_{1}, \ldots, \gamma_{M}\right\}$ be a valid distribution. By

$$
\alpha_{1}+\gamma_{j} \leq 1, j=1, \ldots, M
$$

Adding these $M$ inequalities and dividing by $M$ gives $\alpha_{1}+$ $\frac{\gamma_{1}+\ldots+\gamma_{M}}{M} \leq 1$.

Now, note that every tree is a bipartite graph [4]. Given a tree, suppose the root constitutes layer 1, the children of the root constitute layer 2 and the children of all the nodes in layer $i$ constitute layer $i+1, i=2,3, \ldots$. Not every tree is mean valid; a counterexample is presented in Section VII-A (see Fig. 10). The following result provides a sufficient condition for a tree to be mean valid.

Proposition 3: A tree in which every node in an odd layer has exactly $\kappa$ children is mean valid.

Proof: Let a tree $T$ in which every node in an odd layer has $\kappa$ children be given. Let $N_{j}$ be the total number of nodes in the $j$ 'th layer of $T$ and $N=\sum_{j \text { odd }} N_{j}$.

Let $A_{i}=\left\{a_{i}\right\}, B_{i}=\left\{b_{i, 1}, \ldots, b_{i, \kappa}\right\}$ and $G^{i}$ be a star with center $a_{i}$ and $\kappa$ leaves- the nodes in $B_{i}$. Note that each $G^{i}$ is mean valid by Proposition 2. Also, $G^{i}, i=1, \ldots, N$, satisfy the hypothesis of Lemma 11 with $d=2, I_{1}^{i}=B_{i}, I_{2}^{i}=A_{i}$, $M_{1}^{i}=\kappa, M_{2}^{i}=1 \forall i, \mathbf{M}^{0}=(\kappa, 1)$ and $c_{i}=1 \forall i$. So by Lemma $11, G=G^{1} \cup G^{2} \cup \ldots \cup G^{N}$ is mean valid.

Now, we will obtain $T$ by adding some edges to $G$ as illustrated in Fig. 9. Let the center, $a_{1}$, of $G^{1}$ be the root of $T$. Note that its children are $b_{1,1}, \ldots, b_{1, \kappa}$, the leaves of $G^{1}$. For $j \in\{1, \ldots, \kappa\}$, suppose $b_{1, j}$ has $l_{j}$ children. Join $b_{1, j}$ by an edge to each of the centers of $l_{j}$ stars out of $G^{2}, \ldots, G^{N}$, using a different set of stars for each $j$. Thus, we have obtained the nodes in the first 4 layers of the tree and the edges connecting them. Suppose a node $b$ in layer 4 has $l^{\prime}$ children. Join it to the centers of $l^{\prime}$ stars out of $G^{1}, \ldots, G^{N}$, which have not been used so far. Proceed in this manner to get the tree $T$. Note that there is no edge between two nodes in the same partition of the tree, which is a bipartite graph (see Fig. 9); so the hypothesis of Lemma 10 is satisfied. Hence, $T$ is mean valid by Lemma 10 . 


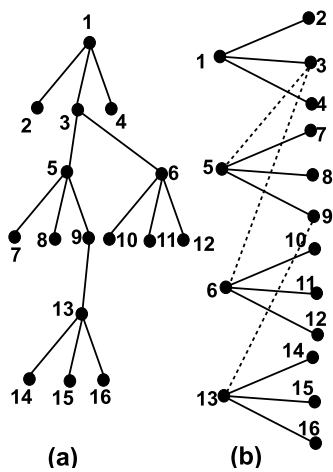

Fig. 9. Part (a) shows a tree in which each node in an odd layer has exactly 3 children. Part (b) shows the construction of the tree. We start with stars with 3 leaves each, whose edges are shown in bold and then add some edges, shown dotted. Note that none of the dotted edges is between two nodes in the same partition of the bipartite graph, so the condition in Lemma 10 is satisfied.

\section{SOME AdDitional Topics}

\section{A. Non Mean Valid Bipartite Graphs}

We have shown in Theorem 2, that a mean valid graph has a unique symmetric NE that has a simple form- under this NE, for every $q \in(0,1)$, each primary offers bandwidth with the same probability $t_{j}$ at all the nodes in each I.S. $I_{j}$, $j \in\{1, \ldots, d\}$. Thus, mean validity is a sufficient condition for an arbitrary graph to have a symmetric NE of the form in Theorem 2 for all values of $q$. The following result shows that for connected bipartite graphs, mean validity is also a necessary condition.

Theorem 3: Let $G$ be a connected bipartite graph that is not mean valid. If $w(q, n)>1-\frac{M_{2}}{M_{1}}$, then $\beta\left(I_{1}\right)=t_{1}, \beta\left(I_{2}\right)=t_{2}$, $\beta(I)=0 \forall I \in \mathscr{I}, I \neq I_{1}, I_{2}$, is not a symmetric NE for any value of $t_{1}$ and $t_{2}$.

Now, we provide an example of a non mean valid bipartite graph and find the symmetric NE and prove its uniqueness. The symmetric NE is not of the form in Theorem 2 for any value of $q \in(0,1)$.

Let the set of nodes be $A \cup B$, where $A=\left\{a_{1}, a_{2}, a_{3}\right\}$ and $B=\left\{b_{1}, b_{2}, b_{3}\right\}$, and let there be an edge between $a_{1}$ (respectively, $b_{1}$ ) and every edge in $B$ (respectively, $A$ ) (see Fig. 10). The only maximal I.S. are $I_{a b}=\left\{a_{2}, a_{3}, b_{2}, b_{3}\right\}$,

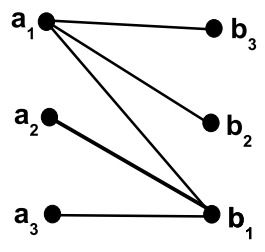

Fig. 10. A non mean valid graph.

$I_{a}=A$ and $I_{b}=B$. The I.S. $I_{a b}$ contains 2 nodes from each of $A$ and $B$, i.e., $m_{1}\left(I_{a b}\right)=m_{2}\left(I_{a b}\right)=2$ in the notation of Lemma 3. Also, $\frac{m_{1}\left(I_{a b}\right)}{3}+\frac{m_{2}\left(I_{a b}\right)}{3}>1$; so (12) is not satisfied. Hence, this is not a mean valid graph by Lemma 3 .

In every symmetric NE, $\beta\left(I_{a}\right)=t_{a}, \beta\left(I_{b}\right)=t_{b}$ and $\beta\left(I_{a b}\right)=1-t_{a}-t_{b}$ for some $0 \leq t_{a}, t_{b} \leq 1$.
Lemma 12: If $w(q, n)>\frac{1}{2}$, then $f_{1}(x)=2 W(1-x)-$ $W(x)$ has a unique root $t_{1} \in[0,1]$. Also, $0<t_{1}<\frac{1}{2}$.

The following theorem provides the symmetric NE in the above graph for each value of $q \in(0,1)$.

Theorem 4: 1) If $w(q, n) \leq \frac{1}{2}$, then the symmetric strategy profile corresponding to $t_{a}=t_{b}=0$ is the unique symmetric NE.

2) If $w(q, n)>\frac{1}{2}$, then the symmetric strategy profile in which $t_{a}=t_{b}=t_{1}$, the root of $f_{1}($.$) , is the unique$ symmetric NE.

Note that $I_{a b}$, which contains 4 nodes, is the largest I.S. So for all values of $q$, primaries offer bandwidth with positive probability at the I.S. $I_{a b}$ in the symmetric NE. Since $I_{a b}$ contains nodes from both $A$ and $B$, the node probabilities at different nodes in $A$ (and $B$ ) are different. Thus, the symmetric $\mathrm{NE}$ is not of the form in Theorem 2 for any value of $q$.

Again, since $I_{a b}$ is the largest I.S., Theorem 4 shows, consistent with intuition, that for small values of $q$, primaries offer bandwith only at $I_{a b}$ with positive probability; when $q$, and thereby the competition at $I_{a b}$, increases beyond a threshold, they also offer bandwidth at $A$ and $B$ with positive probability.

\section{B. Some Remarks}

For simplicity, we have assumed that there are $k$ secondaries at each node, where $k$ is a constant. However, all our results readily generalize to the case in which the number of secondaries at node $v \in V$ is $K_{v}$, where $\left\{K_{v}: v \in V\right\}$ are i.i.d. random variables such that the primaries apriori know only the probability mass function (p.m.f.) for $K_{v}, \operatorname{Pr}\left(K_{v}=k\right)=\gamma_{k}$, but not the values of $\left\{K_{v}: v \in V\right\}$. Let:

$$
w(q, n)=\sum_{k=1}^{n-1} \gamma_{k} \sum_{i=k}^{n-1}\left(\begin{array}{c}
n-1 \\
i
\end{array}\right) q^{i}(1-q)^{n-1-i}
$$

All our results go through if $w(q, n)$ in the above equation is used in place of (2).

Now, in presence of spatial reuse, we would in general have $\mathrm{NE}$ that are not symmetric. For example, suppose there are two nodes $v_{1}$ and $v_{2}$ connected by an edge, two primaries $(n=2)$ and one secondary at each node $(k=1)$. Then the strategy profiles in which primary 1 offers bandwidth at node $v_{1}$ and primary 2 at node $v_{2}$ w.p. 1 , or vice versa, and both primaries set a price of $\nu$ w.p. 1, are NE, apart from the symmetric NE in Theorem 2. This is in contrast with the game at a single location, in which the symmetric NE is the unique NE [20].

\section{NUMERICAL STUDIES}

In this section, we describe numerical computations that are directed towards assessing the impact of price competition among the primaries on the aggregate revenue of the primaries and the affordability of spectrum for the secondaries. Towards that end, we compare the symmetric NE resulting from price competition with a scheme, denoted by OPT, under which all the primaries cooperate so as to maximize the sum of their revenues.

We consider the specific case of a grid graph $\mathcal{H}_{m, m}$, which was introduced in Section IV-B (see Fig. 13). By part 4 of 
Theorem 1, this is a mean valid graph and, in the notation of Definition 3, $d=4$ and the I.S. $I_{1}, I_{2}, I_{3}$ and $I_{4}$ are as described in Section VI-A. We use the parameter values $n=$ $10, k=3, \nu=1, c=0$ and $m=7$.

In $\mathcal{H}_{m, m}$, the symmetric NE is of the form in Theorem 2 and under OPT, the I.S. $I_{1}, \ldots, I_{4}$ are selected in order of size and all the primaries always select the highest price $\nu$.

Fig. 11 reveals, as expected, that price competition significantly reduces the aggregate revenue of the primaries relative to OPT. Also, overall, the ratio between the aggregate revenues under the symmetric NE and under OPT decreases as $q$ increases since the competition increases. Fig. 12 shows that under price competition, the expected price per unit of bandwidth is lower at the nodes in the larger I.S. This is because primaries prefer larger I.S. and hence the competition is more intense there, driving down the prices.

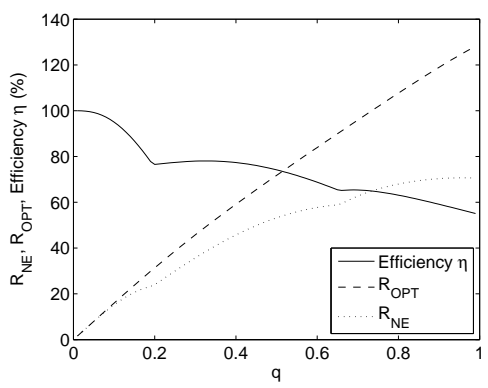

Fig. 11. The figure plots the aggregate revenues of the primaries, $R_{N E}$ and $R_{O P T}$, under the symmetric NE and OPT respectively, and the efficiency of the symmetric NE, $\eta=\frac{R_{N E}}{R_{O P T}}$ (in \%), versus $q$.

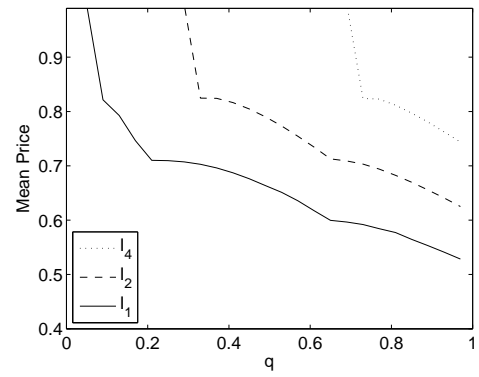

Fig. 12. The figure shows the mean price of bandwidth, given that it is offered, at a (fixed) node in each of $I_{1}, I_{2}$ and $I_{4}$ vs $q$ under the symmetric NE.

\section{Discussion AND Future Work}

For analytical tractability, we have considered the symmetric case where each primary has unused bandwidth with the same probability $q$. Even for price competition at a single location, analysis of the asymmetric case, where the probabilities of bandwidth availability of different primaries are not equal, has been done only in some special cases, e.g., when there is only one secondary [20]. An important problem for future research is the analysis of the asymmetric case in the spatial reuse setting.

\section{APPENDIX}

Let $W(\alpha)$ be as in (7). We will use the following result throughout.

Lemma 13: (i) For $0 \leq \alpha \leq 1,0 \leq W(\alpha)<1$, (ii) $W(0)=1$, and (iii) $W(\alpha)$ is strictly decreasing in $\alpha$.

Lemma 13 follows from (7), the fact that $w(0, n)=0$ and Lemma 1.

\section{A. Proofs of results in Section IV}

Proof of Lemma 3: Suppose $G$ is mean valid. Fix an $I \in \mathscr{I}$. Let

$$
\mathbf{1}_{I}\left(a_{j, l}\right)= \begin{cases}1, & \text { if } a_{j, l} \in I \\ 0, & \text { else }\end{cases}
$$

Consider a distribution $\left\{\alpha_{j, l}: j=1, \ldots, d ; l=1, \ldots, M_{j}\right\}$ in which bandwidth is offered at node $a_{j, l} \in I_{j}$ w.p. $\alpha_{j, l}=$ $\mathbf{1}_{I}\left(a_{j, l}\right)$. This is a valid distribution because it corresponds to the distribution $\left\{\beta(I)=1, \beta\left(I^{\prime}\right)=0 \forall I^{\prime} \in \mathscr{I}, I^{\prime} \neq I\right\}$. Also,

$$
\sum_{l=1}^{M_{j}} \alpha_{j, l}=\sum_{l=1}^{M_{j}} \mathbf{1}_{I}\left(a_{j, l}\right)=m_{j}(I), \quad j=1, \ldots, d
$$

Let $\bar{\alpha}_{j}$ be given by (11). Since the graph is mean valid, (10) holds. Substituting $\sum_{l=1}^{M_{j}} \alpha_{j, l}=m_{j}(I)$ from (26) into (10), we get (12).

To prove the converse, suppose (12) holds. Let $\left\{\alpha_{j, l}: j=\right.$ $\left.1, \ldots, d ; l=1, \ldots, M_{j}\right\}$ be a valid distribution. By definition, there exists a distribution $\{\beta(I): I \in \mathscr{I}\}$ such that:

$$
\alpha_{j, l}=\sum_{I \in \mathscr{I}: a_{j, l} \in I} \beta(I)
$$

which can be written as:

$$
\alpha_{j, l}=\sum_{I \in \mathscr{I}} \beta(I) \mathbf{1}_{I}\left(a_{j, l}\right)
$$

Now,

$$
\begin{aligned}
& \sum_{j=1}^{d}\left(\frac{\sum_{l=1}^{M_{j}} \alpha_{j, l}}{M_{j}}\right) \\
= & \sum_{j=1}^{d} \frac{1}{M_{j}}\left\{\sum_{l=1}^{M_{j}} \sum_{I \in \mathscr{I}} \beta(I) \mathbf{1}_{I}\left(a_{j, l}\right)\right\} \quad \text { by (28)) } \\
= & \sum_{I \in \mathscr{I}} \beta(I)\left\{\sum_{j=1}^{d} \frac{\sum_{l=1}^{M_{j}} \mathbf{1}_{I}\left(a_{j, l}\right)}{M_{j}}\right\} \\
= & \sum_{I \in \mathscr{I}} \beta(I)\left\{\sum_{j=1}^{d} \frac{m_{j}(I)}{M_{j}}\right\} \quad\left(\text { since } \sum_{l=1}^{M_{j}} \mathbf{1}_{I}\left(a_{j, l}\right)=m_{j}(I)\right) \\
\leq & 1 \text { (by (12)) }
\end{aligned}
$$

So (10) holds and hence $G$ is mean valid. 


\section{B. Proofs of results in Section V}

The following lemma is used in the proof of Lemma 4.

Lemma 14: Let $N \geq 2$ be an integer and $\alpha_{1}, \ldots, \alpha_{N}, f_{1}, \ldots, f_{N}$ be real numbers. Then:

$N\left(\sum_{i=1}^{N} \alpha_{i} f_{i}\right)-\left(\sum_{i=1}^{N} \alpha_{i}\right)\left(\sum_{i=1}^{N} f_{i}\right)=\sum_{1 \leq i<j \leq N}\left(\alpha_{j}-\alpha_{i}\right)\left(f_{j}-f_{i}\right)$

Proof: We prove the result by induction. For $N=2$ :

$$
\begin{aligned}
\text { LHS } & =2\left(\alpha_{1} f_{1}+\alpha_{2} f_{2}\right)-\left(\alpha_{1}+\alpha_{2}\right)\left(f_{1}+f_{2}\right) \\
& =\left(\alpha_{2}-\alpha_{1}\right)\left(f_{2}-f_{1}\right) \\
& =\text { RHS }
\end{aligned}
$$

Suppose the result is true for $N$. For $N+1$ :

$$
\begin{aligned}
& L H S=(N+1)\left(\sum_{i=1}^{N} \alpha_{i} f_{i}+\alpha_{N+1} f_{N+1}\right)- \\
& \left(\sum_{i=1}^{N} \alpha_{i}+\alpha_{N+1}\right)\left(\sum_{i=1}^{N} f_{i}+f_{N+1}\right) \\
& =\left\{N\left(\sum_{i=1}^{N} \alpha_{i} f_{i}\right)-\left(\sum_{i=1}^{N} \alpha_{i}\right)\left(\sum_{i=1}^{N} f_{i}\right)\right\} \\
& +N \alpha_{N+1} f_{N+1}+\sum_{i=1}^{N} \alpha_{i} f_{i}+\alpha_{N+1} f_{N+1} \\
& -\alpha_{N+1}\left(\sum_{i=1}^{N} f_{i}\right)-\left(\sum_{i=1}^{N} \alpha_{i}\right) f_{N+1}-\alpha_{N+1} f_{N+1} \\
& =\sum_{1 \leq i<j \leq N}\left(\alpha_{j}-\alpha_{i}\right)\left(f_{j}-f_{i}\right) \\
& +\sum_{i=1}^{N}\left(\alpha_{N+1} f_{N+1}+\alpha_{i} f_{i}-\alpha_{N+1} f_{i}-\alpha_{i} f_{N+1}\right)
\end{aligned}
$$

(by induction hypothesis and collecting terms)

$$
=R H S
$$

The result follows by induction.

Proof of Lemma 4: By symmetry, we can assume WLOG that $\alpha_{1} \leq \alpha_{2} \ldots \leq \alpha_{N}$. Since $f($.$) is strictly decreasing,$ $f\left(\alpha_{1}\right) \geq f\left(\alpha_{2}\right) \geq \ldots \geq f\left(\alpha_{N}\right)$. Now:

$$
\begin{aligned}
& \left(\sum_{i=1}^{N} \alpha_{i} f\left(\alpha_{i}\right)\right)-\bar{\alpha}\left(\sum_{i=1}^{N} f\left(\alpha_{i}\right)\right) \\
& \quad=\frac{1}{N}\left(N\left(\sum_{i=1}^{N} \alpha_{i} f\left(\alpha_{i}\right)\right)-\left(\sum_{i=1}^{N} \alpha_{i}\right)\left(\sum_{i=1}^{N} f\left(\alpha_{i}\right)\right)\right) \\
& \quad=\frac{1}{N} \sum_{1 \leq i<j \leq N}\left(\alpha_{j}-\alpha_{i}\right)\left(f\left(\alpha_{j}\right)-f\left(\alpha_{i}\right)\right) \quad(\text { by }(29)(30)
\end{aligned}
$$

For $i<j, \alpha_{i} \leq \alpha_{j}$ and $f\left(\alpha_{i}\right) \geq f\left(\alpha_{j}\right)$. So each term in (30) is $\leq 0$. Hence, the expression in (30) is 0 iff each term is 0 , which happens iff $\alpha_{1}=\ldots=\alpha_{N}=\bar{\alpha}$.

Proof of Lemma 6: Let

$$
\begin{aligned}
U^{*} & =\max \left\{U_{1}\left(I_{j}\right): j \in\{1, \ldots, d\}\right\} \\
& =\max \left\{M_{j} W\left(t_{j}\right): j \in\{1, \ldots, d\}\right\} \text { (by (19)) }
\end{aligned}
$$

and $B=\left\{j \in\{1, \ldots, d\}: M_{j} W\left(t_{j}\right)=U^{*}\right\}$. Note that $B$ is the set of indices of the I.S. out of $I_{1}, \ldots, I_{d}$ that yield the highest payoff and $U^{*}$ is the value of that payoff.

By definition of $B$ :

$$
\begin{gathered}
W\left(t_{j}\right)=\frac{U^{*}}{M_{j}}, \forall j \in B \\
W\left(t_{j}\right)<\frac{U^{*}}{M_{j}}, \quad \forall j \notin B .
\end{gathered}
$$

Let $I$ be any I.S. containing $m_{j}(I)$ nodes from $I_{j}, j=$ $1, \ldots, d$. By (18):

$$
\begin{aligned}
U_{1}(I) & =\sum_{j=1}^{d} m_{j}(I) W\left(t_{j}\right) \\
& \leq \sum_{j=1}^{d} m_{j}(I)\left(\frac{U^{*}}{M_{j}}\right) \\
& \leq U^{*}(\text { by }(12))
\end{aligned}
$$

So $\max _{I \in \mathscr{I}} U_{1}(I) \leq U^{*}$, and since $U_{1}\left(I_{j}\right)=U^{*}, j \in B$, each $I_{j}, j \in B$, is a best response. Now, for $I$ as defined above, suppose $m_{j}(I) \geq 1$ for some $j \notin B$. Then the inequality in (33) is strict. So $U_{1}(I)<U^{*}$ and $I$ is not a best response. Thus, each $I \in \mathscr{I}$ containing a node from $I_{j}$ for some $j \notin B$ is not a best response. In particular, $\forall j \notin B, I_{j}$ is not a best response and, since primaries offer bandwidth at $I_{j}$ w.p. $t_{j}$ in the above NE, $t_{j}=0$ for all $j \notin B$.

It now suffices to show that $B=\left\{1, \ldots, d^{\prime}\right\}$ for some $1 \leq d^{\prime} \leq d$. Suppose not. Then there exist $j, l \in\{1, \ldots, d\}$ such that $j<l, j \notin B$ and $l \in B$. Since $j \notin B, t_{j}=0$ by the previous paragraph. Now, by (18):

$$
\begin{aligned}
U_{1}\left(I_{j}\right) & =M_{j} W\left(t_{j}\right) \\
& =M_{j}(\text { by part (ii) of Lemma 13) } \\
& \geq M_{l}(\text { by }(9), \text { since } j<l) \\
& \geq M_{l} W\left(t_{l}\right) \quad(\text { by part }(\mathrm{i}) \text { of Lemma 13) } \\
& =U^{*}
\end{aligned}
$$

So $I_{j}$ is a best response, which is a contradiction since $j \notin B$.

Proof of Lemma 8: Suppose primaries $2, \ldots, n$ use the strategy $\psi$, under which bandwidth is offered at the nodes in $I_{j}$ w.p. $t_{j}, j=1, \ldots, d$. By (17) and part (ii) of Lemma 13, $W\left(t_{j}\right)=1, j>d^{\prime}$. So by (18), the payoff of primary 1 if it plays I.S. $I_{j}, j \in\left\{1, \ldots, d^{\prime}\right\}$ (resp., $j \in\left\{d^{\prime}+1, \ldots, d\right\}$ ) is $U_{1}\left(I_{j}\right)=M_{j} W\left(t_{j}\right)$ (resp., $U_{1}\left(I_{j}\right)=M_{j}$ ). Hence, by (20) and (9), for some $U^{*}$,

$$
U^{*}=U_{1}\left(I_{1}\right)=\ldots=U_{1}\left(I_{d^{\prime}}\right)>U_{1}\left(I_{d^{\prime}+1}\right) \geq \ldots \geq U_{1}\left(I_{d}\right) .
$$

The maximum payoff that primary 1 can get at a node $v \in I_{j}$, $j \in\left\{1, \ldots, d^{\prime}\right\}$ equals

$$
W\left(t_{j}\right)=\frac{U_{1}\left(I_{j}\right)}{M_{j}}=\frac{U^{*}}{M_{j}} .
$$

Now, for $j>d^{\prime}, M_{j}=U_{1}\left(I_{j}\right)<U^{*}$. So the maximum payoff that primary 1 can get at a node $v \in I_{j}, j>d^{\prime}$ is

$$
1<\frac{U^{*}}{M_{j}} \text {. }
$$


Now, let $I$ be an I.S. containing $m_{j}(I)$ nodes from $I_{j}, j=$ $1, \ldots, d$. By (34) and (35):

$$
\begin{aligned}
U_{1}(I) & \leq U^{*}\left(\sum_{j=1}^{d} \frac{m_{j}(I)}{M_{j}}\right) \\
& \leq U^{*}(\text { by (12)) }
\end{aligned}
$$

Since $U_{1}\left(I_{1}\right)=\ldots=U_{1}\left(I_{d^{\prime}}\right)=U^{*}, I_{1}, \ldots, I_{d^{\prime}}$ are best responses. Under the strategy $\psi$, primary 1 can only play $I_{1}, \ldots, I_{d^{\prime}}$ with positive probability; hence, $\psi$ is a best response.

Proof of Lemma 9: Existence: For convenience, let $M_{d+1}=0$. Fix $q \in(0,1)$. For $x \in\left[M_{1} W(1), M_{1}\right]$ and $j \in\{1, \ldots, d\}$, if $M_{j} \geq x$, then we show that the equation:

$$
M_{j} W\left(t_{j}\right)=x
$$

has a unique solution $t_{j}(x) \in[0,1]$. Let $h\left(t_{j}\right)=M_{j} W\left(t_{j}\right)$. By part (ii) of Lemma 13, $h(0)=M_{j} \geq x$. Also,

$$
\begin{aligned}
h(1) & =M_{j} W(1) \\
& \leq M_{1} W(1)(\text { by (9)) } \\
& \leq x
\end{aligned}
$$

Also, by (2) and (7), $h\left(t_{j}\right)$ is a continuous function of $t_{j}$. So by the intermediate value theorem [3], $h\left(t_{j}\right)=x$ has a solution in $[0,1]$. By part (iii) of Lemma $13, h\left(t_{j}\right)$ is a strictly decreasing function of $t_{j}$; so this solution, say $t_{j}(x)$, is unique. For $x=M_{j}, t_{j}=0$ satisfies (37) by part (ii) of Lemma 13. So $t_{j}\left(M_{j}\right)=0$.

Since $h\left(t_{j}\right)$ is strictly decreasing on $0 \leq t_{j} \leq 1$, it is invertible. Also, since the inverse of a continuous function is continuous (see Theorem 4.17 in [3]), $h^{-1}(x)$ is continuous. But $x=h\left(t_{j}(x)\right)$. So $t_{j}(x)=h^{-1}(x)$. Thus, $t_{j}(x)$ is continuous in $x$ for $x \leq M_{j}$. For $x>M_{j}$, define $t_{j}(x)=0$. As shown above, $t_{j}\left(M_{j}\right)=0$. So $t_{j}(x)$ is continuous on $\left[M_{1} W(1), M_{1}\right]$. Let,

$$
T(x)=\sum_{j=1}^{d} t_{j}(x)
$$

As shown above, $h\left(t_{j}\right)$ is strictly decreasing on $0 \leq t_{j} \leq 1$ for $j=1, \ldots, d$. So $t_{j}(x)=h^{-1}(x)$ is strictly decreasing for $x \leq M_{j}$. Also, by definition, $t_{j}(x)=0$ on $M_{j}<x \leq M_{1}$. So by (38), $T(x)$ is strictly decreasing on $\left[M_{1} W(1), M_{1}\right]$ (note that $t_{1}(x)$ is strictly decreasing on $\left.x \leq M_{1}\right)$. Also, $t_{j}\left(M_{1}\right)=$ $0, j=1, \ldots, d$. So

$$
T\left(M_{1}\right)=0 .
$$

Now, for $j=1$ and $x=M_{1} W(1), t_{1}=1$ satisfies (37). So $t_{1}\left(M_{1} W(1)\right)=1$ and hence, by (38):

$$
T\left(M_{1} W(1)\right) \geq 1 .
$$

Now, since each $t_{j}(x), j=1, \ldots, d$, is continuous on $\left[M_{1} W(1), M_{1}\right]$, so is $T(x)$ by (38). Hence, by (39), (40) and the intermediate value theorem, the equation $T(x)=1$ has a solution $x^{*} \in\left[M_{1} W(1), M_{1}\right]$, which is unique because $T(x)$ is strictly decreasing. Let $d^{\prime}(q)=\max \left\{j: M_{j} \geq x^{*}\right\}$. By definition of $t_{j}(x)$, for $j=1, \ldots, d^{\prime}(q), M_{j} W\left(t_{j}\left(x^{*}\right)\right)=x^{*}$ and for $j>d^{\prime}(q), M_{j}<x^{*}$ and hence $t_{j}\left(x^{*}\right)=0$.
Thus, $\left(t_{1}\left(x^{*}\right), \ldots, t_{d}\left(x^{*}\right)\right)$ satisfy (17) and (20). Also, by (38), $\sum_{j=1}^{d} t_{j}\left(x^{*}\right)=T\left(x^{*}\right)=1$; so $\left(t_{1}\left(x^{*}\right), \ldots, t_{d}\left(x^{*}\right)\right)$ is a probability distribution. The result follows.

Uniqueness: Fix $q$. We now show the uniqueness of $d^{\prime}(q)$ and the distribution $\left(t_{1}, \ldots, t_{d}\right)$ satisfying (17) and (20). Assume, to reach a contradiction, that there exist $e, f \in$ $\{1, \ldots, d\}$ and probability distributions $t=\left(t_{1}, \ldots, t_{d}\right)$ and $s=\left(s_{1}, \ldots, s_{d}\right)$ such that $t_{j}=0$ (respectively, $s_{j}=0$ ) for $j>e$ (respectively, $j>f$ ) and for some $y$ and $z$ :

$$
\begin{aligned}
& y=M_{1} W\left(t_{1}\right)=\ldots=M_{e} W\left(t_{e}\right)>M_{e+1} \\
& z=M_{1} W\left(s_{1}\right)=\ldots=M_{f} W\left(s_{f}\right)>M_{f+1}
\end{aligned}
$$

First, suppose $e=f$. If $y=z$, then by (41) and (42), $M_{j} W\left(t_{j}\right)=M_{j} W\left(s_{j}\right), j=1, \ldots, e$. By part (iii) of Lemma 13, $W($.$) is a one-to-one function; so t_{j}=s_{j}$, $j=1, \ldots, e$. Also, $t_{j}=s_{j}=0, j>e$. So $t=s$.

Now, suppose $z>y$. Then $M_{j} W\left(s_{j}\right)>M_{j} W\left(t_{j}\right), j=$ $1, \ldots, e$. So $W\left(s_{j}\right)>W\left(t_{j}\right)$, and by part (iii) of Lemma 13 , $s_{j}<t_{j}, j=1, \ldots, e$. So $1=\sum_{j=1}^{e} s_{j}<\sum_{j=1}^{e} t_{j}=1$, which is a contradiction. Thus, $z>y$ is not possible. By symmetry, $z<y$ is also not possible.

Now, suppose $e<f$. Then by (41) and (42), $z=$ $M_{e+1} W\left(s_{e+1}\right) \leq M_{e+1}<y$. So for $j \in\{1, \ldots, e\}$ :

$$
M_{j} W\left(s_{j}\right)=z<y=M_{j} W\left(t_{j}\right)
$$

which implies $s_{j}>t_{j}$. So $\sum_{j=1}^{e} s_{j}>\sum_{j=1}^{e} t_{j}=1$, which is a contradiction. So $e<f$ is not possible. By symmetry, $e>f$ is also not possible. The result follows.

Monotonicity Now, we show that $d^{\prime}(q)$ is an increasing function of $q$. Suppose not. Then there exist $q$ and $q^{\prime}$ such that $q<q^{\prime}, d^{\prime}(q)=e, d^{\prime}\left(q^{\prime}\right)=f$ and $e>f$. Hence, by (20) and (7), there exist probability distributions $\left(t_{1}, \ldots, t_{d}\right)$ and $\left(s_{1}, \ldots, s_{d}\right)$ such that for some $y$ and $z$ :

$y=M_{1}\left(1-w\left(q t_{1}, n\right)\right)=\ldots=M_{e}\left(1-w\left(q t_{e}, n\right)\right)>M_{e+1}$

$z=M_{1}\left(1-w\left(q^{\prime} s_{1}, n\right)\right)=\ldots=M_{f}\left(1-w\left(q^{\prime} s_{f}, n\right)\right)>M_{f+1}$

So

$$
y=M_{f+1}\left(1-w\left(q t_{f+1}, n\right)\right) \leq M_{f+1}<z .
$$

Hence, for $j=1, \ldots, f, M_{j}\left(1-w\left(q t_{j}, n\right)\right)<M_{j}(1-$ $\left.w\left(q^{\prime} s_{j}, n\right)\right)$. So $w\left(q^{\prime} s_{j}, n\right)<w\left(q t_{j}, n\right)$. By Lemma $1, w(x, n)$ is strictly increasing in $x$. So $q^{\prime} s_{j}<q t_{j}$. Since $q<q^{\prime}, t_{j}>s_{j}$. Thus, $\sum_{j=1}^{f} t_{j}>\sum_{j=1}^{f} s_{j}=1$, which contradicts the fact that $\left(t_{1}, \ldots, t_{d}\right)$ is a probability distribution. The result follows.

Finally, we show that $t_{1} \geq t_{2} \ldots \geq t_{d}$. For $1 \leq i<j \leq$ $d^{\prime}(q), M_{i} W\left(t_{i}\right)=M_{j} W\left(t_{j}\right)$ by (20). But $M_{i} \geq M_{j}$ by (9); so $W\left(t_{i}\right) \leq W\left(t_{j}\right)$ and hence, by part (iii) of Lemma 13, $t_{i} \geq t_{j}$. For $l>d^{\prime}(q), t_{l}=0$ by (17). The result follows.

\section{Proofs of results in Section VI}

Proof of Lemma 10: Since no edge in $E^{\prime}$ is between two nodes in the same I.S. $I_{j}$, it follows that in $G^{\prime}, I_{1}, \ldots, I_{d}$ are disjoint maximal I.S. whose union is $V$. Using the notation in Definition 3, let $\left\{\alpha_{j, l}: j=1, \ldots, d ; l=1, \ldots, M_{j}\right\}$ be a valid distribution in $G^{\prime}$. We will show that (10) holds. Then it will follow from Definition 3 that $G^{\prime}$ is mean valid. 
Let $\mathscr{I}_{G^{\prime}}$ (respectively, $\mathscr{I}_{G}$ ) be the set of I.S. in $G^{\prime}$ (respectively, $G$ ). Since $E \subset E^{\prime}$, each I.S. in $G^{\prime}$ is an I.S. in $G$ as well, i.e. $\mathscr{I}_{G^{\prime}} \subset \mathscr{I}_{G}$.

Now, since the distribution $\left\{\alpha_{j, l}\right\}$ is valid in $G^{\prime}$, by definition, there exists a distribution $\left\{\beta^{\prime}(I): I \in \mathscr{I}_{G^{\prime}}\right\}$ such that

$$
\alpha_{v}=\sum_{I \in \mathscr{I}_{G^{\prime}}: v \in I} \beta^{\prime}(I) \forall v \in V .
$$

Define a distribution on $\mathscr{I}_{G}$ as follows:

$$
\beta(I)= \begin{cases}\beta^{\prime}(I) & \text { if } I \in \mathscr{I}_{G^{\prime}} \\ 0 & \text { if } I \in \mathscr{I}_{G} \backslash \mathscr{I}_{G^{\prime}}\end{cases}
$$

By (45) and (46):

$$
\alpha_{v}=\sum_{I \in \mathscr{I}_{G}: v \in I} \beta(I) \forall v \in V .
$$

So by definition, $\left\{\alpha_{i, j}\right\}$ is a valid distribution in $G$ as well. Since $G$ is mean valid, (10) holds, which completes the proof.

Proof of Lemma 11: First, note that $\left\{\left(I_{j}^{1} \cup \ldots \cup I_{j}^{N}\right): j=\right.$ $1, \ldots, d\}$ are disjoint maximal I.S. in $G$; so the first condition in Definition 3 is satisfied.

Let $\left\{\alpha_{j, l}^{i}: j=1, \ldots, d ; l=1, \ldots, M_{j}^{i}\right\}$ be a valid distribution in $G^{i}$. Since $G^{i}$ is mean valid:

$$
\sum_{j=1}^{d}\left(\frac{\sum_{l=1}^{M_{j}^{i}} \alpha_{j, l}^{i}}{M_{j}^{i}}\right) \leq 1, \quad i=1, \ldots, N
$$

Now, it is given that:

$$
M_{j}^{i}=c_{i} M_{j}^{0}, \quad i=1, \ldots, N ; j=1, \ldots, d
$$

Adding (49) over $i=1, \ldots, N$ :

$$
M_{j}^{0}\left(c_{1}+\ldots+c_{N}\right)=M_{j}^{1}+\ldots+M_{j}^{N}, j=1, \ldots, d
$$

Multiplying (48) by $c_{i}$, using (49) and adding over $i=$ $1, \ldots, N$, we get:

$$
\sum_{i=1}^{N} \sum_{j=1}^{d}\left(\frac{\sum_{l=1}^{M_{j}^{i}} \alpha_{j, l}^{i}}{M_{j}^{0}}\right) \leq c_{1}+\ldots+c_{N}
$$

Dividing both sides by $c_{1}+\ldots+c_{N}$ and using (50):

$$
\sum_{j=1}^{d}\left(\frac{\sum_{i=1}^{N} \sum_{l=1}^{M_{j}^{i}} \alpha_{j, l}^{i}}{M_{j}^{1}+\ldots+M_{j}^{N}}\right) \leq 1
$$

So $G$ satisfies the second condition in Definition 3 as well and hence is mean valid.

Proof of part 2 of Theorem 1: In Section VI-A, we showed that $\mathcal{G}_{m}$ is mean valid for even $m$. Now, let $m$ be odd, say $m=2 N-1$ for some integer $N \geq 2$. Consider a valid distribution $\left\{\alpha_{i}: i=1, \ldots, 2 N-1\right\}$, where $\alpha_{i}$ is the probability with which bandwidth is offered at node $v_{i}$. With $I_{1}$ and $I_{2}$ as defined in Section VI-A, note that $\left|I_{1}\right|=N$ and $\left|I_{2}\right|=N-1$. Let

$$
\bar{\alpha}^{1}=\frac{\alpha_{1}+\alpha_{3}+\ldots+\alpha_{2 N-1}}{N}
$$

and

$$
\bar{\alpha}^{2}=\frac{\alpha_{2}+\alpha_{4}+\ldots+\alpha_{2 N-2}}{N-1}
$$

To show that Condition 2 in Definition 3 is satisfied, we need to show that $\bar{\alpha}^{1}+\bar{\alpha}^{2} \leq 1$, i.e.

$$
\begin{aligned}
& (N-1)\left(\alpha_{1}+\alpha_{3}+\ldots+\alpha_{2 N-1}\right) \\
& \quad+\quad N\left(\alpha_{2}+\alpha_{4}+\ldots+\alpha_{2 N-2}\right) \leq N(N-1)
\end{aligned}
$$

Since $\mathcal{G}_{2 N-1}$ is a bipartite graph and the distribution $\left\{\alpha_{i}\right\}$ is valid, the necessary condition in (22) holds and in this case becomes:

$$
\alpha_{i}+\alpha_{i+1} \leq 1, \quad i=1,2, \ldots, 2 N-2
$$

Now,

$$
\begin{aligned}
\text { LHS of } & (51) \\
= & \left\{(N-1)\left(\alpha_{1}+\alpha_{2}\right)+\left(\alpha_{2}+\alpha_{3}\right)\right\} \\
& +\left\{(N-2)\left(\alpha_{3}+\alpha_{4}\right)+2\left(\alpha_{4}+\alpha_{5}\right)\right\} \\
& +\left\{(N-3)\left(\alpha_{5}+\alpha_{6}\right)+3\left(\alpha_{6}+\alpha_{7}\right)\right\} \\
& +\ldots \\
& +\left\{2\left(\alpha_{2 N-5}+\alpha_{2 N-4}\right)+(N-2)\left(\alpha_{2 N-4}+\alpha_{2 N-3}\right)\right\} \\
& +\left\{\left(\alpha_{2 N-3}+\alpha_{2 N-2}\right)+(N-1)\left(\alpha_{2 N-2}+\alpha_{2 N-1}\right)\right\} \\
\leq & \{(N-1)+1\}+\{(N-2)+2\}+\ldots \\
& +\{2+(N-2)\}+\{1+(N-1)\} \quad(\text { by }(52)) \\
= & N(N-1)
\end{aligned}
$$

which proves (51) and the result follows.

Proof of part 4 of Theorem 1: In Section VI-A, we showed that $\mathcal{H}_{m, m}$ is mean valid for even $m$. Now, let $m$ be odd. With $I_{1}, I_{2}, I_{3}$ and $I_{4}$ as defined in Section VI-A, it is easy to check that $\left|I_{1}\right|=\left(\frac{m+1}{2}\right)^{2},\left|I_{2}\right|=\frac{m^{2}-1}{4},\left|I_{3}\right|=\frac{m^{2}-1}{4}$ and $\left|I_{4}\right|=\left(\frac{m-1}{2}\right)^{2}$.

Consider a valid distribution $\left\{\alpha_{z}: z \in V\right\}$, where $\alpha_{z}$ is the probability with which bandwidth is offered at node $z$. We now show that the graph is mean valid by showing that (10) holds, which in this case becomes:

$$
\begin{array}{r}
(m-1)^{2}\left(\sum_{z \in I_{1}} \alpha_{z}\right)+\left(m^{2}-1\right)\left(\sum_{z \in I_{2}} \alpha_{z}\right)+\left(m^{2}-1\right)\left(\sum_{z \in I_{3}} \alpha_{z}\right) \\
+(m+1)^{2}\left(\sum_{z \in I_{4}} \alpha_{z}\right) \leq \frac{\left(m^{2}-1\right)^{2}}{4} .
\end{array}
$$

Consider cliques $C_{i, j}, i, j \in\{0, \ldots, m\}$. For $i, j \in$ $\{1, \ldots, m-1\}, C_{i, j}$ is as defined in Section VI-A. For $i$ or $j$ (or both) equal to 0 or $m$, let $C_{i, j}$ be "dummy cliques", defined for convenience (see Fig. 15). For $i, j \in\{0, \ldots, m\}$ :

$$
\sum_{z \in C_{i j}} \alpha_{z} \leq 1
$$

because, if not, then bandwidth would be offered simultaneously at two or more of the nodes in $C_{i j}$ (which are neighbors) with a positive probability. For $i \in\{0, \ldots, m\}$, let:

$$
e_{i}= \begin{cases}m-i, & i \text { odd } \\ i, & i \text { even }\end{cases}
$$

For $i, j \in\{0, \ldots, m\}$, let

$$
f_{i j}=e_{i} e_{j}
$$


Note that by definition of the cliques $\left\{C_{i, j}\right\}$, node $v_{i j}$ belongs to each of the cliques $C_{i-1, j-1}, C_{i-1, j}, C_{i, j-1}$ and $C_{i, j}$ as shown in Fig. 16. So multiplying (54) by $f_{i j}$ and adding over $i, j \in\{0,1, \ldots, m\}$ gives:

$$
\sum_{z \in V} g_{z} \alpha_{z} \leq g_{0}
$$

where,

$$
g_{v_{i j}}=f_{i-1, j-1}+f_{i-1, j}+f_{i, j-1}+f_{i j}
$$

and

$$
\begin{aligned}
g_{0} & =\sum_{i=0}^{m} \sum_{j=0}^{m} f_{i, j}=\sum_{i=0}^{m} \sum_{j=0}^{m} e_{i} e_{j}=\left(\sum_{i=0}^{m} e_{i}\right)^{2} \\
& =\left(\sum_{i=0, i \text { odd }}^{m}(m-i)+\sum_{i=0, i \text { even }}^{m} i\right)^{2}=\frac{\left(m^{2}-1\right)^{2}}{4}(59)
\end{aligned}
$$

We will show below that

$$
g_{z}= \begin{cases}(m-1)^{2}, & z \in I_{1} \\ \left(m^{2}-1\right), & z \in I_{2} \text { or } z \in I_{3} \\ (m+1)^{2}, & z \in I_{4}\end{cases}
$$

Note that (53) follows from (57), (59) and (60), which shows that $\mathcal{H}_{m, m}$ is mean valid.

Now we show (60). By definition of the I.S. $I_{1}, I_{2}, I_{3}$ and $I_{4}$ (see Section VI-A), for $v_{i j} \in I_{1}, i$ and $j$ are odd, for $v_{i j} \in I_{2}$, $i$ is odd and $j$ is even, for $v_{i j} \in I_{3}, i$ is even and $j$ is odd and for $v_{i j} \in I_{4}, i$ and $j$ are even. So for $v_{i j} \in I_{1}$, by (55), (56) and (58):

$$
\begin{aligned}
g_{v_{i j}}= & (i-1)(j-1)+(i-1)(m-j)+(m-i)(j-1) \\
& +(m-i)(m-j) \\
= & (m-1)^{2}
\end{aligned}
$$

Similarly, for $v_{i j} \in I_{2}$ :

$$
\begin{aligned}
g_{v_{i j}}= & (i-1)(m-j+1)+(i-1) j+(m-i)(m-j+1) \\
& +(m-i) j \\
= & m^{2}-1
\end{aligned}
$$

For $v_{i j} \in I_{3}, g_{v_{i j}}=m^{2}-1$ by symmetry with the case $v_{i j} \in I_{2}$. For $v_{i j} \in I_{4}$ :

$$
\begin{aligned}
g_{v_{i j}}= & (m-i+1)(m-j+1)+(m-i+1) j \\
& +i(m-j+1)+i j \\
= & (m+1)^{2}
\end{aligned}
$$

Thus, we have shown (60), which completes the proof.

Proof of part 5 of Theorem 1: In Section VI-A, we considered the case $m$ even. The proof of the fact that $\mathcal{T}_{m, m, m}$ is mean valid for $m$ odd is similar to that for $\mathcal{H}_{m, m}$ with $m$ odd; we outline the differences. We define the cliques $C_{i j l}$, $i, j, l \in\{0,1, \ldots, m\}$, similar to $C_{i j}$ for the case $\mathcal{H}_{m, m}$. Consider a valid distribution $\left\{\alpha_{z}: z \in V\right\}$. Then similar to (54), we get:

$$
\sum_{z \in C_{i j l}} \alpha_{z} \leq 1
$$

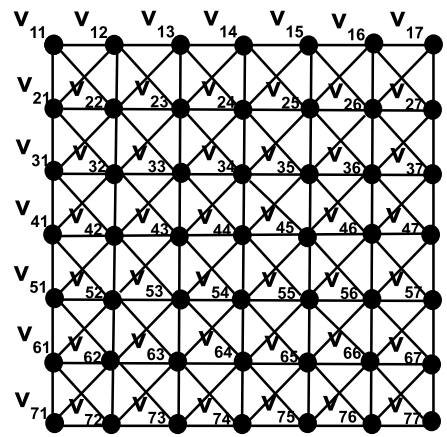

Fig. 13. The figure shows a grid graph $\mathcal{H}_{m, m}$ with $m=7$.

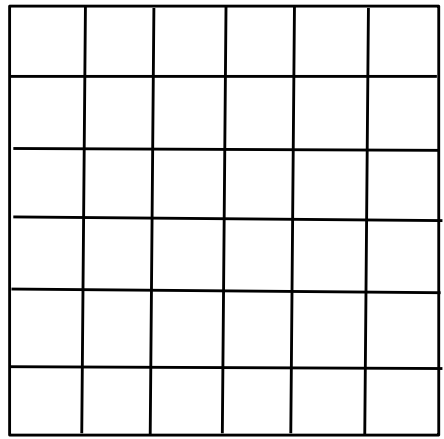

Fig. 14. The figure shows a tiling of a plane with squares, e.g. cells in a cellular network. Transmissions at neighboring cells interfere with each other. The corresponding conflict graph is $\mathcal{H}_{6,6}$.

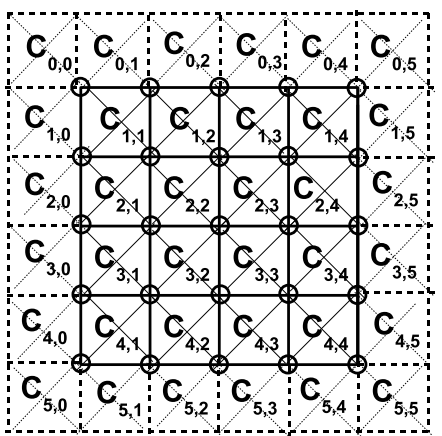

Fig. 15. The figure shows the cliques in $\mathcal{H}_{5,5}$. The cliques with dotted outlines are the dummy cliques.

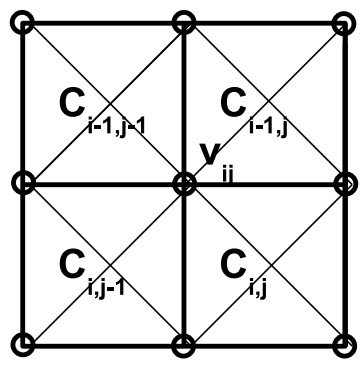

Fig. 16. The node $v_{i j}$ and the cliques $C_{i-1, j-1}, C_{i-1, j}, C_{i, j-1}$ and $C_{i, j}$.

Let $e_{i}$ be as in (55) and $f_{i j l}=e_{i} e_{j} e_{l}, i, j, l \in$ $\{0, \ldots, m\}$. Multiplying (61) by $f_{i j l}$ and adding over $i, j, l \in$ $\{0,1, \ldots, m\}$, we get (57) for some numbers $\left\{g_{z}: z \in V\right\}$ and $g_{0}$. Now, node $v_{i j l}$ is at the center of the cliques $C_{i-1, j-1, l-1}$, 
$C_{i-1, j-1, l}, C_{i-1, j, l-1}, C_{i-1, j, l}, C_{i, j-1, l-1}, C_{i, j-1, l}, C_{i, j, l-1}$, and $C_{i, j, l}$. Using this fact, $g_{v_{i j l}}$ for $v_{i j l}$ in each of $I_{1}, \ldots, I_{8}$ can be computed similar to the derivation of (60). Also, $g_{0}$ can be calculated similar to (59). Substituting these values of $\left\{g_{z}: z \in V\right\}$ and $g_{0}$ into (57), we get (10) for $\mathcal{T}_{m, m, m}$ and thereby the mean validity follows from Definition 3 .

\section{Proofs of results in Section VII-A}

Proof of Theorem 3: Suppose $\beta\left(I_{1}\right)=t_{1}, \beta\left(I_{2}\right)=t_{2}$, where $t_{1}+t_{2}=1$ is a symmetric NE. By (18):

$$
U_{1}\left(I_{j}\right)=M_{j} W\left(t_{j}\right), j=1,2 .
$$

First, suppose $t_{1}=0, t_{2}=1$. Since $\beta\left(I_{2}\right)=t_{2}>0$, $I_{2}$ is a best response. By (62) and part (ii) of Lemma 13, $U_{1}\left(I_{1}\right)=M_{1}$. Again, by (62), and since $W(1)<1$ by part (i) of Lemma 13:

$$
U_{1}\left(I_{2}\right)=M_{2} W(1)<M_{2} \leq M_{1}=U_{1}\left(I_{1}\right)
$$

which contradicts the fact that $I_{2}$ is a best response. So $t_{1}=$ $0, t_{2}=1$ is not a symmetric NE.

Now, suppose $t_{1}=1, t_{2}=0$. Then $I_{1}$ is a best response. Similar to the previous paragraph, $U_{1}\left(I_{1}\right)=M_{1} W(1)$ and $U_{1}\left(I_{2}\right)=M_{2}$. So by (7):

$$
U_{1}\left(I_{1}\right)-U_{1}\left(I_{2}\right)=M_{1}\left(1-\frac{M_{2}}{M_{1}}-w(q, n)\right)<0
$$

since $w(q, n)>1-\frac{M_{2}}{M_{1}}$. This contradicts the fact that $I_{1}$ is a best response. Thus, $t_{1}=1, t_{2}=0$ is not a symmetric NE.

Suppose $0<t_{1}, t_{2}<1$. Let $I \in \mathscr{I}$ be such that:

$$
\frac{m_{1}(I)}{M_{1}}+\frac{m_{2}(I)}{M_{2}}>1,
$$

which exists by Lemma 3 since $G$ is not mean valid. Since $\beta\left(I_{1}\right), \beta\left(I_{2}\right)>0, I_{1}$ and $I_{2}$ are best responses. So $U_{1}\left(I_{1}\right)=$ $U_{1}\left(I_{2}\right)=U^{*}$, where $U^{*}$ is the maximum payoff of any I.S. By (62):

$$
W\left(t_{j}\right)=\frac{U^{*}}{M_{j}}, \quad j=1,2
$$

Now, by (18):

$$
\begin{aligned}
U_{1}(I) & =m_{1}(I) W\left(t_{1}\right)+m_{2}(I) W\left(t_{2}\right) \\
& =U^{*}\left(\frac{m_{1}(I)}{M_{1}}+\frac{m_{2}(I)}{M_{2}}\right)(\text { by }(64)) \\
& >U^{*}(\text { by }(63))
\end{aligned}
$$

which contradicts the fact that $U^{*}$ is the maximum payoff of any I.S.

Proof of Lemma 12: By (2) and (7), $W($.$) is a continuous$ function. So $f_{1}(x)$ is continuous on $[0,1]$. Also, $w^{\prime}(x, n)>$ $0 \forall x \in(0,1)$ [19]. So by (7), $W^{\prime}(\alpha)<0 \forall \alpha \in(0,1)$. Hence, for $x \in(0,1)$ :

$$
f_{1}^{\prime}(x)=-2 W^{\prime}(1-x)-W^{\prime}(x)>0 .
$$

So $f_{1}(x)$ is strictly increasing on $[0,1]$ [3].

Also, by (7), $f_{1}(0)=2 W(1)-W(0)=1-2 w(q, n)<0$ since $w(q, n)>\frac{1}{2}$, and $f_{1}\left(\frac{1}{2}\right)=W\left(\frac{1}{2}\right)=1-w\left(\frac{q}{2}, n\right)>0$. So by the intermediate value theorem [3], $f_{1}(x)$ has a root $t_{1} \in\left(0, \frac{1}{2}\right)$. Also, $t_{1}$ is the unique root in $[0,1]$ since $f_{1}(x)$ is strictly increasing.
1) Proof of Theorem 4: Consider a symmetric strategy profile under which each primary offers bandwidth at $I_{a}$ (respectively, $I_{b}$ ) w.p. $t_{a}$ (respectively, $t_{b}$ ) and at $I_{a b}$ w.p. $1-t_{a}-t_{b}$. By (6), the corresponding node probabilities are $\alpha_{a_{1}}=t_{a}, \alpha_{a_{2}}=\alpha_{a_{3}}=1-t_{b}, \alpha_{b_{1}}=t_{b}, \alpha_{b_{2}}=\alpha_{b_{3}}=1-t_{a}$. So by (18), the total expected payoffs of primary 1 if it offers bandwidth at each of the three I.S. are:

$$
\begin{gathered}
U_{1}\left(I_{a b}\right)=2 W\left(1-t_{b}\right)+2 W\left(1-t_{a}\right) \\
U_{1}\left(I_{a}\right)=2 W\left(1-t_{b}\right)+W\left(t_{a}\right) \\
U_{1}\left(I_{b}\right)=W\left(t_{b}\right)+2 W\left(1-t_{a}\right)
\end{gathered}
$$

Intuitively, since $I_{a b}$ is the largest I.S., we expect that in a symmetric NE, primaries would not offer bandwidth at one or both of $I_{a}$ and $I_{b}$ without offering it at $I_{a b}$. The following result confirms this.

Lemma 15: Let $q \in(0,1)$ be arbitrary. None of the following can hold in a symmetric NE: (i) $t_{a}=1$, (ii) $t_{b}=1$ (iii) $0<t_{a}, t_{b}<1$ and $t_{a}+t_{b}=1$.

Proof: First, suppose $t_{a}=1$ in a symmetric NE. Since $t_{a}>0, I_{a}$ is a best response. Also, $t_{b}=0$. So by (65), (66), (7) and the fact that $w(0, n)=0$ :

$$
U_{1}\left(I_{a b}\right)-U_{1}\left(I_{a}\right)=1+w(q, n)>0
$$

So $U_{1}\left(I_{a b}\right)>U_{1}\left(I_{a}\right)$, which contradicts the fact that $I_{a}$ is a best response. Thus, $t_{a}=1$ is not possible. By symmetry, $t_{b}=1$ is also not possible.

Now, suppose $0<t_{a}, t_{b}<1$ and $t_{a}+t_{b}=1$. Since $t_{a}, t_{b}>$ $0, I_{a}$ and $I_{b}$ are best responses. So $U_{1}\left(I_{a}\right)=U_{1}\left(I_{b}\right)$. By (66), (67), (7) and the fact that $t_{a}+t_{b}=1$, we get $w\left(q t_{a}, n\right)=$ $w\left(q t_{b}, n\right)$. So by Lemma $1, t_{a}=t_{b}=\frac{1}{2}$. Hence, by (65), (66) and (7):

$$
U_{1}\left(I_{a b}\right)-U_{1}\left(I_{a}\right)=\left(1-w\left(\frac{q}{2}, n\right)\right)>0
$$

which contradicts the fact that $I_{a}$ is a best response.

Now we are ready to prove Theorem 4.

Case 1: $w(q, n) \leq \frac{1}{2}$. Let $t_{a}$ and $t_{b}$ be arbitrary. Вy (65), (66) and (7):

$$
\begin{aligned}
& U_{1}\left(I_{a b}\right)-U_{1}\left(I_{a}\right) \\
= & 1-2 w\left(q\left(1-t_{a}\right), n\right)+w\left(q t_{a}, n\right) \\
\geq & 1-2 w(q, n)(\text { by Lemma } 1) \\
\geq & 0\left(\text { since } w(q, n) \leq \frac{1}{2}\right)
\end{aligned}
$$

Note that if $t_{a}>0$, then the inequality in (68) is strict. So $U_{1}\left(I_{a b}\right)>U_{1}\left(I_{a}\right)$, which is a contradiction because $t_{a}>0$ implies that $I_{a}$ is a best response. Hence, $t_{a}=0$. By symmetry, $t_{b}=0$. If $t_{a}=t_{b}=0$, then $U_{1}\left(I_{a b}\right) \geq U_{1}\left(I_{a}\right)$ and $U_{1}\left(I_{a b}\right) \geq U_{1}\left(I_{b}\right)$; so $I_{a b}$ is a best response, which is consistent with the fact that it is played w.p. 1. Thus, $t_{a}=t_{b}=0$ is the unique symmetric NE.

Case 2: $w(q, n)>\frac{1}{2}$. By Lemma $15, t_{a}+t_{b}<1$ for every symmetric NE and hence $I_{a b}$ is a best response. Now, suppose $t_{a}=0$. By (65), (66), (7) and the fact that $w(0, n)=0$, we get $U_{1}\left(I_{a b}\right)-U_{1}\left(I_{a}\right)=1-2 w(q, n)<0$ since $w(q, n)>\frac{1}{2}$. So $U_{1}\left(I_{a}\right)>U_{1}\left(I_{a b}\right)$, which contradicts the fact that $I_{a b}$ is a best response. Hence, $t_{a}>0$. By symmetry, $t_{b}>0$. 
Thus, all three of $I_{a}, I_{b}$ and $I_{a b}$ are best responses. So $U_{1}\left(I_{a b}\right)=U_{1}\left(I_{a}\right)=U_{1}\left(I_{b}\right)$. Substituting (65), (66) and (67), these are satisfied iff $t_{a}=t_{b}=t_{1}$, the root of $f_{1}(x)=0$. This completes the proof.

\section{REFERENCES}

[1] I. Akyildiz, W.-Y. Lee, M. Vuran, S. Mohanty "NeXt generation/dynamic spectrum access/cognitive radio wireless networks: a survey”. In Comp. Networks, Vol. 50, 13, pp. 2127-59, 2006.

[2] R. Myerson, "Game Theory: Analysis of Conflict", Harvard University Press, 1997.

[3] W. Rudin, "Principles of Mathematical Analysis”, Mc-Graw Hill, Third Edition, 1976.

[4] D. West, Introduction to Graph Theory, 2nd ed., Prentice Hall, 2000.

[5] A. Mas-Colell, M. Whinston, J. Green, "Microeconomic Theory", Oxford University Press, 1995.

[6] M. J. Osborne, and C. Pitchik, "Price Competition in a CapacityConstrained Duopoly", In Journal of Economic Theory, 38(2), pp. 238260, 1986

[7] D.M. Kreps, J.A. Scheinkman, "Quantity Precommitment and Bertrand Competition Yield Cournot Outcomes", In Bell Journal of Economics, 14, pp. 326-337, Autumn 1983.

[8] M. Janssen, E. Rasmusen "Bertrand Competition Under Uncertainty", In Journal of Industrial Economics, 50(1): pp. 11-21, March 2002.

[9] S.-F. Cheng, D.M. Reeves, Y. Vorobeychik, M.P. Wellman, "Notes on Equilibria in Symmetric Games", In AAMAS-04 Workshop on GameTheoretic and Decision-Theoretic Agents, 2004.

[10] C. Courcoubetis and R. Weber, "Pricing Communication Networks", John Wiley \& Sons, Ltd. 2003.

[11] O. Ileri, D. Samardzija, T. Sizer, N. B. Mandayam, "Demand Responsive Pricing and Competitive Spectrum Allocation via a Spectrum Policy Server", In Proc. of IEEE DySpan, 2005.

[12] P. Maille, B. Tuffin "Analysis of Price Competition in a Slotted Resource Allocation Game", In Proc. of Infocom, 2008.

[13] P. Maille, B. Tuffin, "Price War with Partial Spectrum Sharing for Competitive Wireless Service Providers", In Proc. of IEEE Globecom, Dec. 2009.

[14] Y. Xing, R. Chandramouli, C. Cordeiro, "Price Dynamics in Competitive Agile Spectrum Access Markets", In IEEE JSAC, Vol. 25, No. 3, April 2007.

[15] D. Niyato, E. Hossain, "Competitive Pricing for Spectrum Sharing in Cognitive Radio Networks: Dynamic Game, Inefficiency of Nash Equilibrium, and Collusion", IEEE JSAC, Vol. 26, No. 1, 2008.

[16] D. Niyato, E. Hossain, Z. Han, "Dynamics of Multiple-Seller and Multiple-Buyer Spectrum Trading in Cognitive Radio Networks: A Game-Theoretic Modeling Approach", IEEE TMC, Vol. 8, No. 8, pp. 1009-1022, Aug. 2009.

[17] X. Zhou, H. Zheng, "TRUST: A General Framework for Truthful Double Spectrum Auctions", In Proc. of Infocom, April 2009.

[18] S. Chawla and T. Roughgarden, "Bertrand Competition in Networks", In Symposium on Algorithmic Game Theory, May, 2008.

[19] G.S. Kasbekar, S. Sarkar, "Spectrum Pricing Games with Bandwidth Uncertainty and Spatial Reuse in Cognitive Radio Networks", in Proc. of MobiHoc, 2010. (Technical report with all proofs available at: http://www.seas.upenn.edu/ swati/publication.htm)

[20] G.S. Kasbekar, S. Sarkar, "Pricing Games under Uncertain Competition", Technical report, 2010. Available at: http://www.seas.upenn.edu/ swati/publication.htm)

[21] G.S. Kasbekar, S. Sarkar, "Spectrum Pricing Games with Spatial Reuse in Cognitive Radio Networks", Technical report, 2010. Available at: http://www.seas.upenn.edu/ swati/publication.htm) 